\title{
Geochemistry of Sarmatian volcanic rocks in the Tokaj Mts (NE Hungary) and their relationship to hydrothermal mineralization
}

\author{
Péter Kiss* \\ Department of Mineralogy \\ Eötvös Loránd University, Budapest
}

Ferenc Molnár

Department of Mineralogy

Eötvös Loránd University, Budapest

\author{
Katalin Gméling \\ Institute of Isotopes \\ Hungarian Academy of Sciences, Budapest
}

\author{
Zoltán Pécskay \\ Institute of Nuclear Research of the \\ Hungarian Academy of Sciences, Debrecen
}

\begin{abstract}
In the Tokaj Mts (NE Hungary), which is a part of the Inner Carpathian Volcanic Arc, large amounts of intermediate-acidic calc-alkaline volcanic rocks accumulated in a $\mathrm{N}-\mathrm{S}$ oriented graben-like structure during the Badenian-Sarmatian-Pannonian period, in relation with the closure of the Alpine Tethys (Penninic) ocean. Although previous research on volcanism and related hydrothermal processes produced a huge number of K/Ar age data no systematic petrochemical database has been available up to now from the Tokaj Mts. In this study we publish new results of geochemical analyses completed on systematically collected basaltic, andesitic, dacitic and rhyolitic rocks, and of the spatialtemporal evaluation of petrochemical signatures, with special reference to origin of magmatism and relationships of rhyolite to hydrothermal mineralization. In the southern Tokaj Mts rhyolite contains K-feldspar phenocrysts, while this phenomenon is absent in the rhyolite from the northern areas of the mountains. In accordance with this, significant potassium enrichment occurs in the south (whole rock $\mathrm{K}_{2} \mathrm{O}$ content varies between 4.35 and $5.61 \mathrm{wt} \%$ ), whereas rhyolite from the northern Tokaj Mts is less enriched in potassium $\left(\mathrm{K}_{2} \mathrm{O}\right.$ content is from 3.28 to $\left.5.1 \mathrm{wt} \%\right)$. The most significant difference between the northern and southern dacite is the age of their formation. They were formed at the same time as rhyolite and andesite (between 13.4 and $11 \mathrm{Ma}$ ) in the northern Tokaj Mts, while they are much younger (10.57-10.1 Ma) in the southern Tokaj Mts, where they post-date hydrothermal activity. The boron content (10.1-52.12 $\mu \mathrm{g} / \mathrm{g})$ and the patterns of other trace elements of the volcanic rocks show typical subduction-related features; however, direct influx of subduction-related fluids during magma generation can be excluded. A more plausible explanation for the magma genesis is decompression melting of a previously metasomatized mantle, enriched with subduction-related components. Additionally, the unmineralized northern rhyolite samples contain much less $\mathrm{Cl}$ (usually below $0.2 \mathrm{wt} \%$ ) than the high-K rhyolite in the southern part of the Tokaj Mts (usually more than 0.2
\end{abstract}


$w t \%)$, which correlates with the presence/absence of spatially and temporally related epithermal mineralization in these areas.

Key words: Tokaj Mts, geochemistry, K/Ar age determination, decompression melting, altered oceanic crust

\section{Introduction}

The Tokaj Mts have a long research history which already began in the late 18th century. In the 19th century important publications had already been presented about the rock types, their alterations and the structure of the mountains; many of them contain very detailed petrographic descriptions (e.g. Szabó 1873, 1881; Szádeczky 1897). In the early 20th century researchers carried out comprehensive observations on the volcanology, eruption styles and rock types occurring in the mountains (Pálfy 1915, 1916). After the first quarter of the 20th century, more detailed research in the different parts of the mountains became the main subject of the studies (e.g. Simkó 1927; Liffa 1928; Pálfy 1935). In the middle of the 20th century many papers were published but were not part of a systematic monitoring, which was later carried out by the Geological Institute of Hungary during the 1960s and the 1970s. This systematic research produced both detailed and comprehensive geologic maps of the area, and along with this the first results of geochemical analysis of different types of rocks were published. Two extended works published results of the above-mentioned research; one focused on andesitic and dacitic rocks (Gyarmati 1977) and the other on rhyolitic rocks (Ilkeiné 1972). An important paper dealing with the structure of the mountains (Zelenka 1963) and a monograph on the ore district at Telkibánya (Széky-Fux 1970), were also published in this period. Another important step was the introduction of K/Ar age determination on volcanic rocks of Hungary (Balogh 1974, 1985; Pécskay et al. 1986), which produced an important database for volcanological reconstruction and also for research on hydrothermal systems (Molnár et al. 1999, Pécskay and Molnár 2002). On the other hand, satellite images and aerial photography, together with geophysical observations, resulted in relevant new conclusions about the structure and volcanology of the mountains (Horváth et al. 1989). The origin of volcanism in the Tokaj Mts was studied in comprehensive papers written about the volcanism of the Western Carpathian region, and Lexa and Konecny (1998) suggested that it occurred due to the decompression melting of an earlier metasomatized mantle wedge in a subduction setting; however, they (and other authors like Downes et al. 1995 or Seghedi et al. 2004) worked from a limited geochemical data base with respect to the Tokaj Mts.

In this study we analyzed samples from earlier described and new locations to establish a geochemical database, which can be useful for observing some petrochemical differences of volcanic rocks. Another important goal was the examination of the geochemical characteristics of rhyolite occurring, both in time and space, close to hydrothermal centers of the Tokaj Mts. 


\section{Geology of the area}

The Tokaj Mts are a part of the Inner Carpathian Volcanic Arc. This arc contains Badenian to almost recently active intermediate-acidic volcanic fields (Pécskay et al. 1995, 2006) exposed from the Central Slovakia Volcanic Field in the Western Carpathians to the Harghita Mts in the Eastern Carpathians. The formation of the calk-alkaline volcanic arc is connected to complex tectonic processes. During the Miocene the suspected oblique southwestward and westward subduction of the remnant Carpathian Flysch Basin began, and it is still active in the Vrancea Zone in the Eastern Carpathians (Horváth 1988; Horváth et al. 1988; Csontos 1995; Nemcok et al. 1998). Parallel to this subduction process a possible slab roll-back effect and crust-thinning was also active and these two factors together generated most of the volcanic activity in the Carpathian-Pannonian Region (CPR) (Szabó et al. 1992).

In the Tokaj Mts volcanism occurred during the Late Badenian-Early Pannonian period (15-9.4 Ma; Pécskay et al. 1995) and produced mainly intermediate-acidic calc-alkaline rocks with lesser amounts of mafic volcanics at the termination of volcanism. The basement is built up by Proterozoic to Mesozoic metamorphic and carbonate rocks, which subsided to form a north-south oriented graben-like structure, which hosts the volcanic sequences of the Tokaj Mts (Gyarmati 1977). The graben is bounded by the Hernád Fault on the western side, the Szamos Fault on the northeastern side, and the Bodrog Fault on the southeastern side (Fig. 1). There are many extension-related faults within the graben, which control the location of volcanic and hydrothermal centers (Pentelényi 1972; Gyarmati 1977). The most important structures are the followings: andesitic lines: (1) between Tokaj and Abaújszántó, (2) between Tolcsva and Gönc; rhyolitic lines: (3) between Sátoraljaújhely and Felsőregmec, (4) between Telkibánya and Makkoshotyka and (5) between Gönc and Abaújszántó (Fig. 1). Results of K/Ar age determinations confirmed that on the scale of the entire mountain range the andesitic and rhyolitic volcanic centers were active at a same time; however, at local scale there are some differences in timing of intermediate and acidic eruptions (Pécskay et al. 1986; Pécskay and Molnár 2002)

\section{Materials and methods}

\section{Sampling}

For both the K/Ar age determination and the geochemical analysis fresh rock samples were selected. In addition we used data from Tonarirni et al. (2001), Ishikawa and Tera (1997), and Harangi et al. (2007) to compare the geochemical characteristics of the Tokaj Mts with other areas (from the CPR and other parts of the world) where decompression melting generated calc-alkaline volcanism. All of these areas are known as volcanic fields generated by decompression melting 


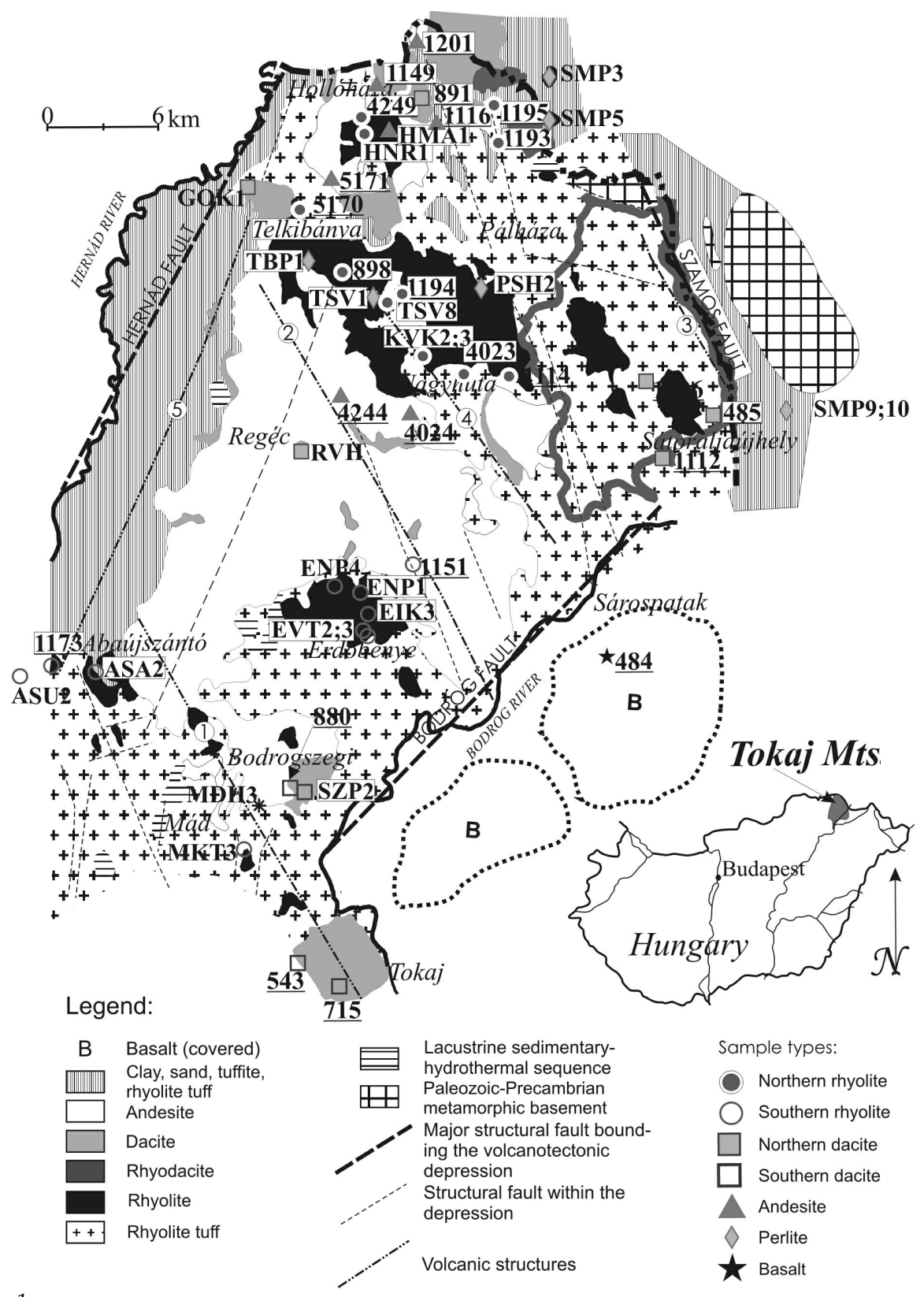

Fig. 1

Geological sketch map of the Tokaj Mts with localities of samples used in this study. The volcanic structures, described in the text, are marked with numbers from 1 to 5 . Inside the bold curve are the badenian rocks 
which affected an earlier metasomatized mantle wedge. The metasomatizing fluid escaped from the subducting slab in each area; therefore we believe that the geochemical characteristics of the rocks from these areas are suitable for comparison with the data from the Tokaj Mts.

$\mathrm{K} / \mathrm{Ar}$ age determinations on various rock types of the Tokaj Mts and their chronological interpretations were previously carried out by Pécskay et al. (1986, 1987, 1995), Balogh et al. (1983), Pécskay and Molnár (2002) and Németh et al. (2006). Some samples from these earlier studies were also included in our current studies.

The sample set of our studies includes one basalt and several andesite, dacite and rhyolite samples from exposures as well as from boreholes. The types of samples are listed in Table 1 and their localities are shown in Fig. 1. Most of the samples are fresh rocks; however, sample MDH-3 is a highly altered kaoliniticsilicic rhyolite from an extrusive dome. This sample was used for the purpose of observing the change of rock composition through hydrothermal alteration.

\section{Analytical techniques}

Prompt gamma activation analysis (PGAA) is especially useful to analyze boron, hydrogen, chlorine and gadolinium concentrations in whole rocks, but all major elements and some other trace elements (like Sc, Co, Ni, Nd and Sm) can also be determined. In contrast to other analytical methods no sample preparation is necessary; hence contamination problems can be avoided (Anderson and Kasztovszky 2004). The accuracy of major and trace element analyses by PGAA has been verified by measurements of geologic reference materials (Gméling et al. 2005). Using PGAA hydrogen can be measured with high sensitivity; thus the $\mathrm{H}_{2} \mathrm{O}$ content of the whole rock could also be

Table 1

Type of occurrences of the samples collected in the Tokaj Mts

\begin{tabular}{|c|c|c|c|c|c|c|c|}
\hline Borehole & \multicolumn{3}{|c|}{ Extrusive dome } & \multicolumn{3}{c|}{ Lava flow } & Altered \\
\hline & andesite & dacite & rhyolite & andesite & dacite & rhyolite & \\
\hline 484, & 1116, & 485, & 898, & 4024, & 543, & 1173, & MDH-3 \\
1149, & 1201 & 880, & 1114, & 4244, & 715, & 1194, & \\
1151 & HMA-1 & 891, & 1193, & 5171 & 1206, & 1195, & \\
& & 1112, & 4023, & & SZP-2 & 5170, & \\
& & EIK-3, & 4249, & & & ASU-2, & \\
& & GOK-1, & MKT-3, & & & EVT-2, & \\
& SMP-4 & KVK-2, & & & EVT-3, & \\
& & TBP-1, & & & KVK-3, & \\
& & TSV-1, & & & PSH-2, & \\
& & TSV-8, & & & ENP-1, & \\
& & PVB-2, & & & HNR-1 & \\
& & SMP-3, & & & & \\
& & & SMP-5, & & & & \\
& & & SMP-9, & & & & \\
& & & SMP-10 & & &
\end{tabular}


determined. The measurements were performed by PGAA at the Budapest Research Reactor (BRR) at the KFKI Research campus (Hungary). The samples were irradiated in a cold neutron beam with a flux of $1.2 \times 10^{8} \mathrm{~cm}^{-2} \mathrm{~s}^{-1}$. The crosssection of the neutron beam during the measurements was collimated to $1 \times 1$ $\mathrm{cm}^{2}$. All the samples were thinner than $5 \mathrm{~mm}$; thus the corrections for the selfabsorption of neutrons and $\gamma$-photons were negligible. The irradiation time was 60-90 minutes. The measurement times were set to achieve acceptable accuracies for the major components. The emitted gamma radiation was detected with a High Purity Germanium detector, surrounded by a bismuth germanate scintillator; the signals were processed with a multichannel analyzer. The measurement system has been described by Belgya and Révay (2004). The spectra were fitted using Hypermet-PC software; element identification was performed using our prompt-gamma library (Révay et al. 2001) and evaluated with an Excel macro developed by Révay (2009). The B data have the smallest relative uncertainty $(1 \%)$ of all elements with a detection limit as low as $0.3 \mu \mathrm{g} / \mathrm{g}$. The relative uncertainties of the major oxides typically range between 1 and 3\%.

Neutron activation analysis (NAA) represents a quantitative as well as qualitative method of high efficiency for the precise determination of concentrations of more than 40 major and trace elements. The determinations were done, using both short and long-term neutron irradiation, at the Budapest Research Reactor (BRR) Hungary at the KFKI Research campus. Fe, Au, as well as $\mathrm{Zr}$ standards were used to check the accuracy of measurements. For short-term irradiation the samples were packed in a polyethylene capsule and irradiated for 120 seconds. After irradiation the samples were measured three times: immediately after irradiation for 15 minutes; after 6 hours for about 10 minutes, and a day later for 10 minutes, respectively, using an HPGe detector with energy resolution of $1.3 \mathrm{keV}$ and relative efficiency of $36 \%$. For long-term irradiation the samples were packed with internal standards, placed in an aluminum container and irradiated for 24 hours. Spectra of induced gamma-activity were recorded after 6, 12 and 60 days of cooling, respectively. The accuracy of the measurement varied between 1 and 10\% depending on the concentration of the element and the sample matrix.

Most of the trace element contents of the samples were determined using a double-focusing inductively coupled plasma mass spectrometer (ICP-MS, ELEMENT2; Thermo Corporation, Bremen, Germany), together with a laser ablation sampler, at the Institute of Isotopes of the Hungarian Academy of Sciences at the KFKI Research campus (Hungary). The samples were finepowdered and mixed with a wax at a 10:1 ratio; $6 \mathrm{~mm}$ wide and $2-3 \mathrm{~mm}$ high pellets were pressed from each of them. NIST-612 glass standard was used during the measurements, and for internal standard the Ca concentration measured with other methods were used. The diameter of the laser was set to $60 \mu \mathrm{m}$, and the strength at $70 \%$. Argon was used as a transport gas. Three lines were evaporated with a laser from every sample pellet at a speed of $5 \mu \mathrm{m} / \mathrm{s}$, for 150 seconds, while background measurement took 50 seconds. 
In this study K/Ar dating was carried out on whole rock samples on the basis of petrology and mineralogical composition of the rock samples. The radiogenic argon content was measured with a magnetic mass spectrometer incorporating an argon extraction line developed in the Institute of Nuclear Research of the Hungarian Academy of Sciences (ATOMKI, Debrecen). K content was measured with a digital flame photometer. Li internal standard and Na buffer was added to the dissolved sample (see details on the experimental method in Balogh 1985). Atomic constants suggested by Steiger and Jägger (1977) were used for calculating the K/Ar age. All analytical errors represent 1 s $(68 \%$ analytical confidence level).

\section{Results}

Petrography

Rhyolite

Rhyolite appears in four well-defined areas of the Tokaj Mountains. In the northern part, two areas are characterized by voluminous rhyolite volcanism; these are located northeast and southeast of Telkibánya (Fig. 1). In the southern part one large rhyolitic area occurs north of Erdóbénye, whereas in the vicinity of Mád many small rhyolite domes and lava flows are present. Early studies (e.g. Ilkeyné 1972) mentioned a remarkable distinction between rhyolite from the northern and from the southern parts of the Tokaj Mts. The "northern rhyolite" does not contain K-feldspar as a phenocryst, whereas the "southern rhyolite" always contain sanidine among the common plagioclase, quartz and (less frequently) biotite (Fig. 2A-B). The phenocrysts in these rocks rarely reach $1 \mathrm{~cm}$ in diameter. The different compositions of the phenocrysts resulted in differences not only in the petrography but also in the geochemistry of the rocks; thus, the southern rhyolite has a higher $\mathrm{K}_{2} \mathrm{O}$ content in contrast with the northern one. On the basis of the airborne K-anomaly map (Molnár et al. 1999) the highest $\mathrm{K}_{2} \mathrm{O}$ contents (above $5 \mathrm{wt} . \%$ ) occur in rhyolite samples collected in the vicinity of Mád. On the other hand, airborne Th-anomalies (up to $25 \mathrm{ppm}$ ) in the Tokaj Mts also correlate well with exposures of rhyolite in the southern Tokaj Mts.

The rhyolitic rocks can also be divided into four subgroups based on the texture of their groundmass: 1) perlite (in perlite there are no real phenocrysts, with the exception of the sample from Pálháza), 2) fluidal rhyolite, 3) spherulitic rhyolite and 4) rhyolite without any special texture in the groundmass. The groundmass is built up from volcanic glass in every sample, but in the northern part of the Tokaj Mts it shows minor recrystallization or dehydration.

\section{Dacite}

Dacite occurs mainly in the form of single domes and related lava flows in the Tokaj Mts. Similarly to rhyolite, dacitic rocks can be distinguished on the basis of their locality, e.g. "northern" and "southern" dacite; however, the basis of this 

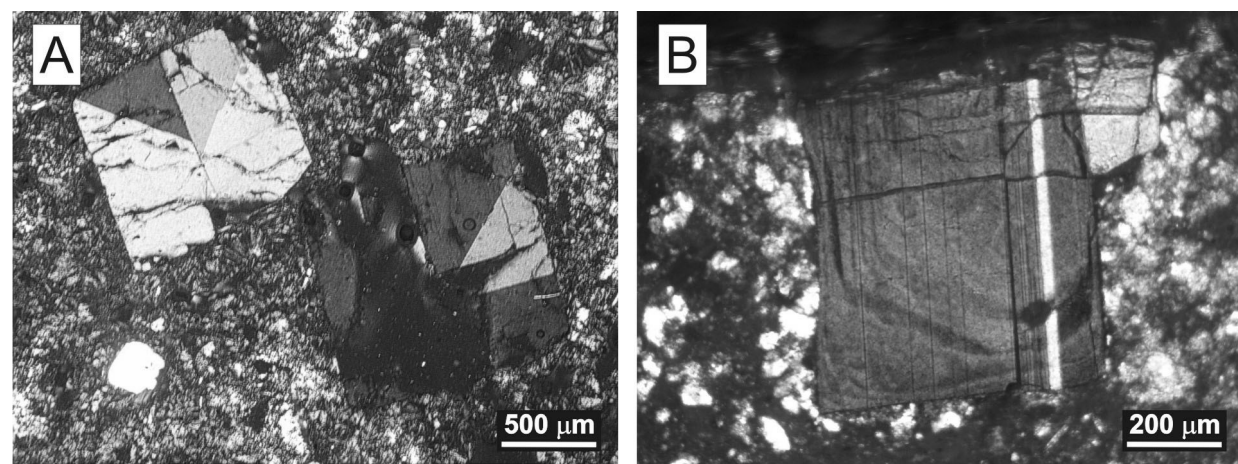

Fig. 2

Microphotograph of A) K-feldspar phenocrysts from a typical southern rhyolite sample (MKT-3, $\times 40$,

$+\mathrm{N}), \mathrm{B}$ ) plagioclase phenocryst from a rock sample collected from the northern Tokaj Mts. (KVK-2, $\times 100,+\mathrm{N})$

discrimination is their age: 11.5-12.9 Ma in the northern part and 10.1 and 10.6 $\mathrm{Ma}$ in the southern part of the Tokaj Mts (Table 1). The main petrographic differences between rhyolite and dacite are the absence in dacite of quartz phenocrysts and (with the exception of the dacite dome at Regéc) biotite, and the presence of plagioclase laths in the glassy groundmass of the dacite. Presence of amphibole (and rarely pyroxene) phenocrysts was also observed in the dacite samples. The occurrence of volcanic rock inclusions is also a characteristic feature of the dacite from the northern Tokaj Mts. The texture of the rock inclusions is holocrystalline, and they contain amphiboles and plagioclase.

Andesite

Almost the entire western part of the Tokaj Mts is built up by andesite and "acidic pyroxene-andesite" (Gyarmati 1977). Fresh andesite was collected from areas located near hydrothermal centers in the vicinity of Telkibánya and Regéc. The andesite does not show local variations, as do rhyolite or dacite. Plagioclase, pyroxene and amphibole are the most common phenocrysts, whereas biotite occurs in a few samples only. The phenocrysts sometimes form aggregates in the groundmass of the andesite. The only similarity to the dacite is the presence of plagioclase laths in the groundmass. Xenoliths of igneous rocks also occur in andesite and they appear very similar to the xenoliths found in some dacite samples; however, they contain more amphibole and plagioclase than the phenocrysts in the host rocks.

\section{Geochemistry}

Major element composition

Our sample set ranges from basalt to rhyolite; therefore the $\mathrm{SiO}_{2}$ content of the samples varies between 50 and $81 \mathrm{wt}$.\%. As usual in calc-alkaline volcanic series 
the $\mathrm{TiO}_{2}, \mathrm{Fe}_{2} \mathrm{O}_{3}$ (total), $\mathrm{MnO}, \mathrm{MgO}$, and $\mathrm{CaO}$ contents of the rocks show negative correlation with the $\mathrm{SiO}_{2}$ content (Fig. 3, Table 2). The $\mathrm{Al}_{2} \mathrm{O}_{3}$ content of the rocks (0.35 to 9.47 wt.\%) increases with the decrease of $\mathrm{SiO}_{2}$ content until $\sim 65$ wt.\%; below this point it appears to be roughly constant but shows some variability in the rocks. The $\mathrm{Na}_{2} \mathrm{O}$ contents vary between 1.68 and $4.88 \mathrm{wt} \%$ (Table 2) and show positive correlation with $\mathrm{SiO}_{2}$ in the case of rocks with basaltic to dacitic composition, whereas in the case of rhyolite (and also perlite) they show high variability. The $\mathrm{K}_{2} \mathrm{O}$ contents also correlate positively with $\mathrm{SiO}_{2}$ and range between 1.39 and $5.62 \mathrm{wt} \%$ (Table 2). Therefore our samples fall in the medium to high- $\mathrm{K}$ series on the $\mathrm{SiO}_{2}$ vs. $\mathrm{K}_{2} \mathrm{O}$ diagram (Fig. 3A). As expected from the petrographic evaluation, rhyolite from the southern Tokaj Mts has a higher $\mathrm{K}_{2} \mathrm{O}$ content compared to the northern type. On the TAS diagram (Fig. 3B), only a few dacite samples fall near the boundary of the dacite and trachyte field; the other samples show clear calc-alkaline properties.

\section{Trace elements}

The boron content varies between 10.1 and $52.12 \mu \mathrm{g} / \mathrm{g}$ (Table 1,2). The boron contents of rocks correlate positively with the $\mathrm{SiO}_{2}$ content (Fig. 3K). However, perlite and most of the northern rhyolite show slightly lower boron content. The origin of the positive correlation between $\mathrm{B}$ and $\mathrm{K}_{2} \mathrm{O}$ (Fig. $3 \mathrm{~K}$ ) is also clear because both are incompatible and fluid-mobile elements.

In contrast with the boron content, chlorine does not show any statistical changes with $\mathrm{SiO}_{2}$ or $\mathrm{K}_{2} \mathrm{O}$ contents of the rocks (Fig. 3L, N). It seems that perlite has the highest $\mathrm{Cl}$ content (0.5-0.7 wt.\% with the exception of sample PSH-2) and southern rhyolite shows higher chlorine content $(0.15-0.6 \mathrm{wt} . \%)$ than the nonperlitic rhyolite (0.04-0.4 wt.\%) in the northern Tokaj Mts. Furthermore, the variation of the chlorine content is much larger with high $\mathrm{SiO}_{2}$ and $\mathrm{K}_{2} \mathrm{O}$ contents. Figure $3 \mathrm{P}$ shows that, except for perlite, there is a weak positive correlation between $\mathrm{B}$ and $\mathrm{Cl}$. However, with exception of $\mathrm{Na}_{2} \mathrm{O}$ there is no correlation between $\mathrm{Cl}$ and any other major or trace elements with which boron shows correlations.

On the multi-element diagrams (Fig. 4) the chondrite-normalized REE pattern shows enrichment, which is hundredfold in the case of LREE, while it decreases down to 10 times more at the HREE. The Eu content of the rocks varies between 0.03 and $1.79 \mu \mathrm{g} / \mathrm{g}$, which causes well-defined negative anomalies, increasing mainly in rhyolite samples, although in some dacite it can also be relevant. The NMORB-normalized diagram (Fig. 4B) shows a similar pattern as the subduction-related volcanic rocks, where the most incompatible (and fluidmobile) trace elements show hundred to thousand fold enrichment (up to $\sim 3000$ fold in the case of $U$ ), while the less incompatible trace elements show minor enrichment compared to NMORB. In this diagram well-defined negative anomalies occur for $\mathrm{Nb}, \mathrm{Sr}$, and $\mathrm{Eu}$; less well-expressed negative anomalies can be seen for $\mathrm{Ba}$ and $\mathrm{Lu}$. Positive anomalies also occur for $\mathrm{U}$ and $\mathrm{K}$, as well as $\mathrm{Pb}$ and 

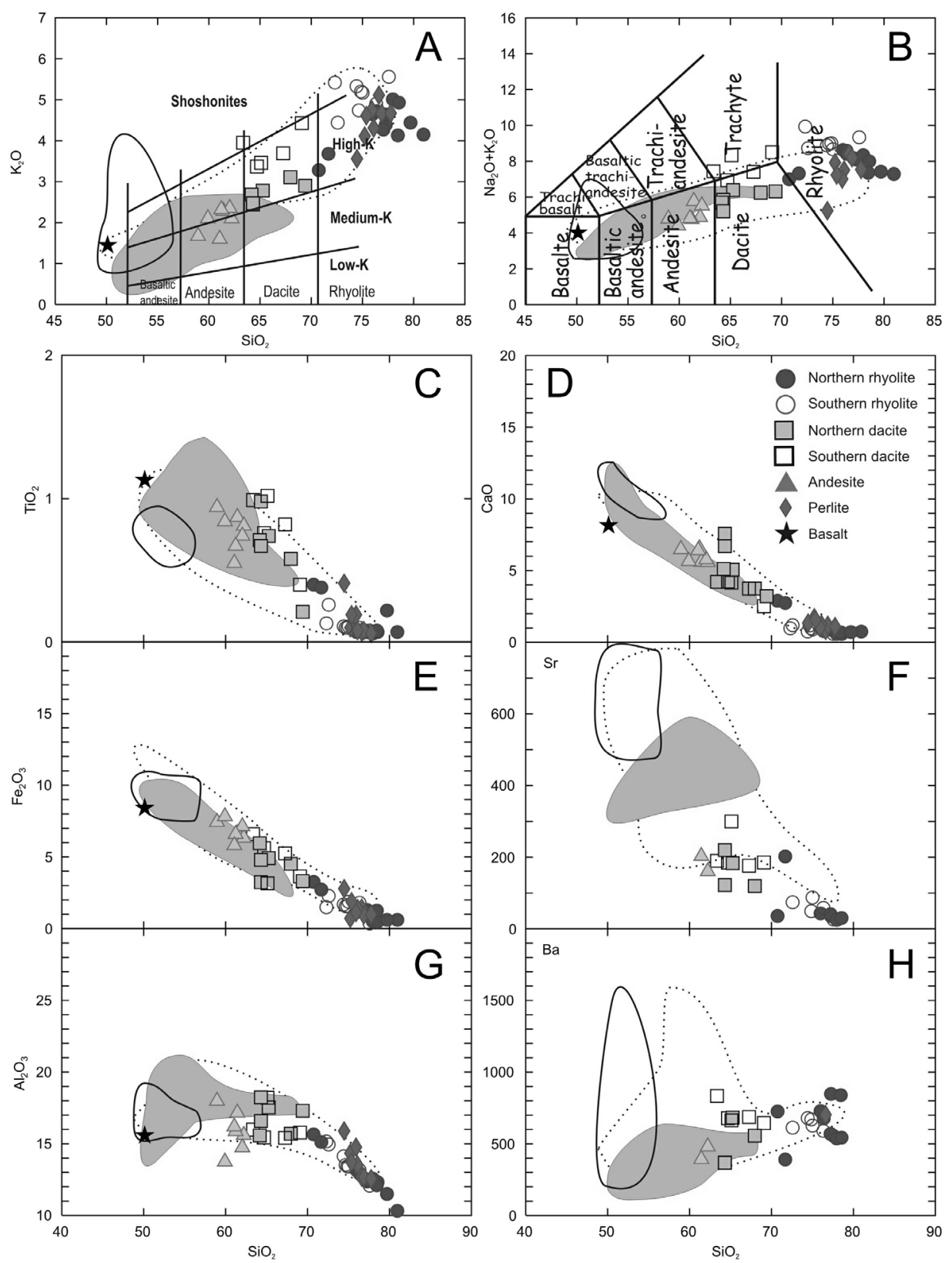

Fig. 3

TAS diagram and variation of selected major and trace elements as a function of the $\mathrm{SiO}_{2}$, (and continued on next page) $\mathrm{B}$ and $\mathrm{Cl}$ content. The configurations represent the values of the comparing areas: thick line: Tonarini et al. (2001); dotted line: Harangi et al. (2007); shaded: Ishikawa and Tera (1997) 
Geochemistry of Sarmatian volcanic rocks in the Tokaj Mts (NE-Hungary) 387
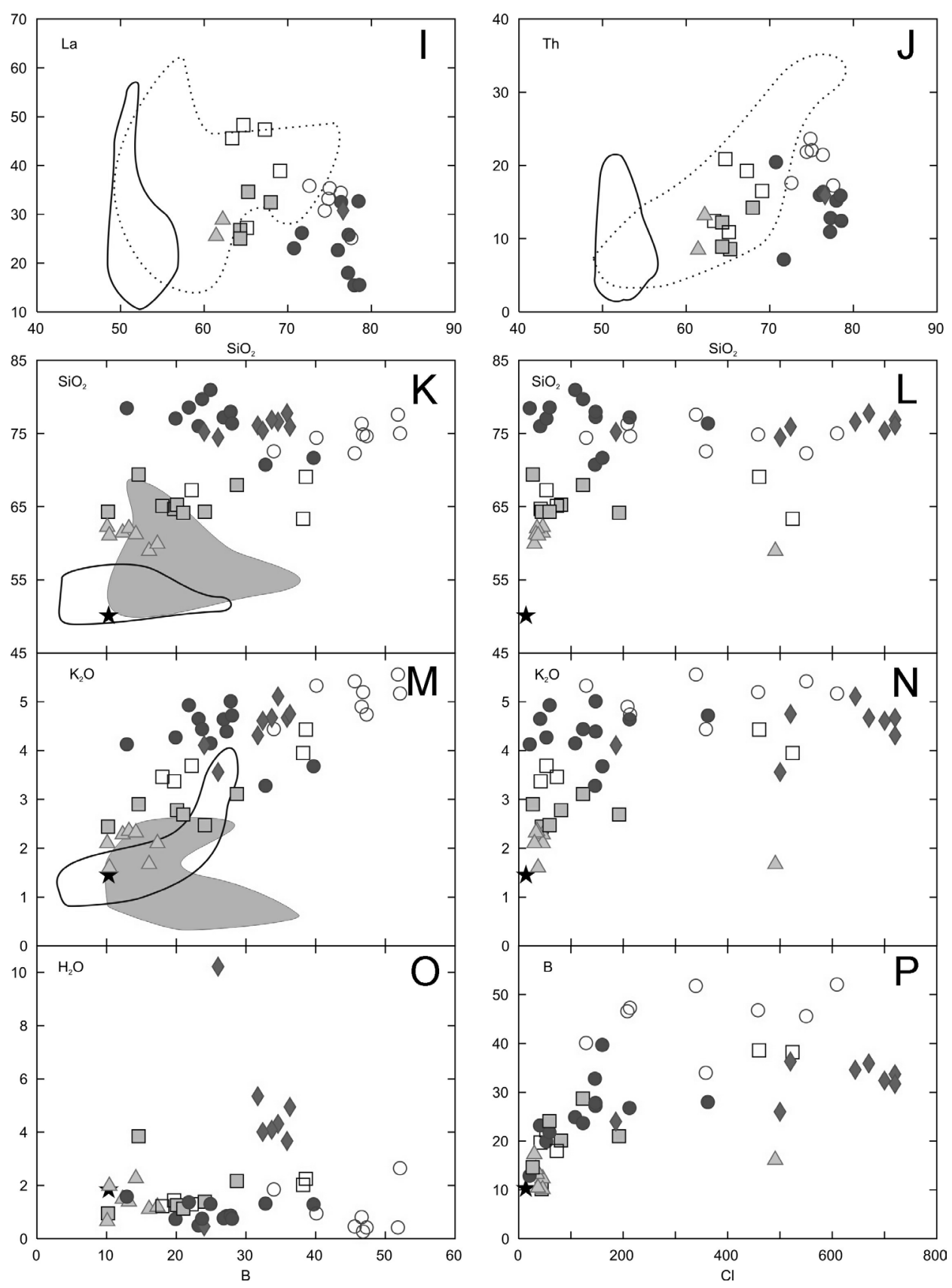

Fig. 3 (cont.) 
Table 2

Major and trace element composition of the Tokaj Mts

\begin{tabular}{|c|c|c|c|c|c|c|c|c|}
\hline Samples & 898 & 1114 & 1193 & 1194 & 1195 & 4023 & 4249 & 5170 \\
\hline Type & N-rhyolite & N-rhyolite & N-rhyolite & N-rhyolite & N-rhyolite & $\mathrm{N}$-rhyolite & N-rhyolite & N-rhyolite \\
\hline Location & $\begin{array}{c}\text { Telki- } \\
\text { bánya, } \\
\text { Susulya- } \\
\text { peak }\end{array}$ & $\begin{array}{c}\text { Kőkapu, } \\
\text { Kemence- } \\
\text { creek }\end{array}$ & $\begin{array}{l}\text { Puszta- } \\
\text { falu, } \\
\text { Bába-hill }\end{array}$ & $\begin{array}{c}\text { Nagy- } \\
\text { bózsva, } \\
\text { Fekete-hill }\end{array}$ & $\begin{array}{l}\text { Füzér- } \\
\text { kajata, } \\
\text { Hársas- } \\
\text { hill }\end{array}$ & $\begin{array}{l}\text { Óhuta, } \\
\text { Pap-hill }\end{array}$ & $\begin{array}{c}\text { Holló- } \\
\text { háza, } \\
\text { Ördögvár }\end{array}$ & $\begin{array}{c}\text { Telki- } \\
\text { bánya, } \\
\text { Nagy- } \\
\text { valley }\end{array}$ \\
\hline Age & $11.2^{* *}$ & $12.1^{* \star}$ & $11.2^{* *}$ & $12.4^{* *}$ & $11.4^{* *}$ & $12.5^{+}$ & $13.44^{+}$ & $13.11^{+}$ \\
\hline $\begin{array}{l}(w t \%) \\
\mathrm{SiO}_{2}\end{array}$ & 77.27 & 75.99 & 70.74 & 77.04 & 71.68 & 77.21 & 77.97 & 78.55 \\
\hline $\mathrm{TiO}_{2}$ & 0.07 & 0.07 & 0.40 & 0.08 & 0.38 & 0.07 & 0.06 & 0.07 \\
\hline $\mathrm{Al}_{2} \mathrm{O}_{3}$ & 12.37 & 13.15 & 15.65 & 12.56 & 15.12 & 12.51 & 12.56 & 12.32 \\
\hline $\mathrm{Fe}_{2} \mathrm{O}_{3}$ & 1.30 & 1.13 & 3.26 & 1.23 & 2.72 & 1.17 & 0.50 & 0.48 \\
\hline $\mathrm{MnO}$ & 0.02 & 0.01 & 0.06 & 0.02 & 0.03 & 0.01 & 0.01 & 0.01 \\
\hline $\mathrm{MgO}$ & nd & nd & nd & $\mathrm{nd}$ & nd & nd & nd & nd \\
\hline $\mathrm{CaO}$ & 0.88 & 1.02 & 2.89 & 0.90 & 2.74 & 0.91 & 0.57 & 0.57 \\
\hline $\mathrm{Na}_{2} \mathrm{O}$ & 3.71 & 3.98 & 3.72 & 3.91 & 3.64 & 3.48 & 3.33 & 3.07 \\
\hline $\mathrm{K}_{2} \mathrm{O}$ & 4.39 & 4.65 & 3.28 & 4.27 & 3.68 & 4.64 & 5.01 & 4.93 \\
\hline $\mathrm{H}_{2} \mathrm{O}$ & 0.85 & 0.50 & 1.32 & 0.74 & 1.29 & 0.77 & 0.87 & 1.37 \\
\hline $\begin{array}{c}(\mu \mathrm{g} / \mathrm{g}) \\
B\end{array}$ & 27.18 & 23.21 & 32.78 & 19.95 & 39.67 & 26.85 & 27.79 & 21.80 \\
\hline $\mathrm{Cl}$ & 146.53 & 41.19 & 146.26 & 52.65 & 160.15 & 211.67 & 146.64 & 58.89 \\
\hline $\mathrm{Nd}$ & 42.04 & 39.93 & bd & 46.77 & 36.24 & 32.01 & 39.86 & 25.41 \\
\hline $\mathrm{Sm}$ & 4.98 & 3.84 & 4.66 & 4.76 & 4.38 & 3.51 & 4.19 & 3.88 \\
\hline $\mathrm{Gd}$ & 6.91 & 4.95 & 5.98 & 5.96 & 5.49 & 4.47 & 5.43 & 5.55 \\
\hline Co & 13.1 & 0.8 & 0.9 & & 13.4 & 1.9 & 0.3 & 0.4 \\
\hline $\mathrm{Ni}$ & 5.2 & $\mathrm{bd}$ & 1.6 & & 0.3 & 95.1 & 1.5 & 1.2 \\
\hline $\mathrm{Cr}$ & 18.1 & 6.0 & 8.6 & & 22.2 & 19.0 & 3.9 & 10.7 \\
\hline V & 88.7 & 4.7 & 3.8 & & 106.5 & 8.2 & 3.7 & 9.2 \\
\hline $\mathrm{Pb}$ & 27.4 & 51.4 & 60.0 & & 25.8 & 35.6 & 50.1 & 35.0 \\
\hline $\mathrm{Sr}$ & 183.3 & 43.4 & 36.3 & & 202.3 & 41.0 & 25.4 & 30.6 \\
\hline $\mathrm{Rb}$ & 161.9 & 268.4 & 288.0 & & 96.5 & 215.1 & 224.1 & 206.9 \\
\hline $\mathrm{Ba}$ & 682.5 & 728.6 & 725.6 & & 391.1 & 569.4 & 537.3 & 543.7 \\
\hline $\mathrm{Zr}$ & 96.3 & 47.8 & 59.5 & & 131.9 & 39.3 & 54.0 & 56.0 \\
\hline $\mathrm{Nb}$ & 11.3 & 15.7 & 19.8 & & 11.9 & 11.2 & 14.3 & 10.8 \\
\hline Th & 8.6 & 15.9 & 20.5 & & 7.2 & 10.9 & 15.2 & 12.4 \\
\hline $\mathrm{Y}$ & 33.3 & 28.3 & 33.4 & & 26.2 & 24.8 & 36.4 & 25.0 \\
\hline $\mathrm{La}$ & 34.6 & 22.6 & 23.0 & & 26.2 & 18.0 & 15.4 & 15.5 \\
\hline $\mathrm{Ce}$ & 85.0 & 58.8 & 53.8 & & 60.3 & 46.5 & 40.0 & 39.6 \\
\hline $\mathrm{Pr}$ & 6.5 & 5.5 & 5.9 & & 7.1 & 4.3 & 4.0 & 4.1 \\
\hline $\mathrm{Eu}$ & 0.4 & 0.4 & 0.4 & & 1.1 & 0.3 & 0.3 & 0.3 \\
\hline $\mathrm{Gd}$ & 4.8 & 4.5 & 5.8 & & 5.7 & 3.2 & 4.2 & 4.3 \\
\hline Dy & 5.2 & 4.9 & 5.3 & & 5.3 & 3.5 & 4.5 & 4.5 \\
\hline $\mathrm{Ho}$ & 1.2 & 1.0 & 1.1 & & 1.0 & 0.6 & 1.0 & 0.9 \\
\hline $\mathrm{Er}$ & 3.3 & 3.0 & 3.3 & & 3.3 & 2.2 & 3.3 & 2.6 \\
\hline $\mathrm{Yb}$ & 3.8 & 3.7 & 4.0 & & 2.9 & 1.8 & 4.7 & 2.5 \\
\hline $\mathrm{Lu}$ & 0.5 & 0.4 & 0.5 & & 0.6 & 0.3 & 0.4 & 0.4 \\
\hline $\mathrm{Li}$ & $\mathrm{bd}$ & $\mathrm{bd}$ & $\mathrm{bd}$ & & 45.5 & 2.5 & 0.7 & nd \\
\hline $\mathrm{Tb}$ & 0.8 & 0.8 & 1.1 & & 0.8 & 0.5 & 0.7 & 0.8 \\
\hline $\mathrm{Tm}$ & 0.5 & 0.4 & 0.7 & & 0.5 & 0.4 & 0.5 & 0.4 \\
\hline $\mathrm{Hf}$ & 2.1 & 2.5 & 2.7 & & 4.1 & 2.2 & 2.5 & 2.7 \\
\hline $\mathrm{Ta}$ & 0.9 & 1.2 & 1.3 & & 0.8 & 0.9 & 1.1 & 0.9 \\
\hline$U$ & 21.3 & 6.0 & 7.4 & & $\mathrm{bd}$ & 107.7 & 6.9 & 10.2 \\
\hline
\end{tabular}


Table 2 cont.

\begin{tabular}{|c|c|c|c|c|c|c|c|c|}
\hline Samples & KVK-2 & KVK-3 & TBP-1 & TSV-1 & TSV-8 & PSH-2 & PVB-2 & HNR-1 \\
\hline Type & \begin{tabular}{|l|} 
N-rhyolite \\
\end{tabular} & \begin{tabular}{|l|} 
N-rhyolite \\
\end{tabular} & perlite & perlite & rhyolite & perlite & perlite & N-rhyolite \\
\hline Location & $\begin{array}{l}\text { Kishuta, } \\
\text { Vörös- } \\
\text { stone }\end{array}$ & $\begin{array}{l}\text { Kishuta, } \\
\text { Vörös- } \\
\text { stone }\end{array}$ & \begin{tabular}{|c|} 
Telkibá- \\
nya, Cser- \\
hill
\end{tabular} & $\begin{array}{c}\text { Telki- } \\
\text { bánya, } \\
\text { Köves- } \\
\text { senyö }\end{array}$ & $\begin{array}{l}\text { Telki- } \\
\text { bánya, } \\
\text { Berenás- } \\
\text { stone }\end{array}$ & $\begin{array}{l}\text { Pálháza, } \\
\text { Sulyom- } \\
\text { hill }\end{array}$ & $\begin{array}{l}\text { Pálháza, } \\
\text { Vadász- } \\
\text { hill }\end{array}$ & $\begin{array}{l}\text { Holló- } \\
\text { háza, } \\
\begin{array}{c}\text { Nagy Pál- } \\
\text { hill }\end{array}\end{array}$ \\
\hline Age & $12.38^{+}$ & $12.55^{+}$ & $11.66^{+}$ & nd & nd & nd & nd & nd \\
\hline $\begin{array}{l}(w t \%) \\
\mathrm{SiO}_{2}\end{array}$ & 76.38 & 78.46 & 76.61 & 76.88 & 80.96 & 75.22 & 77.76 & 79.70 \\
\hline $\mathrm{TiO}_{2}$ & 0.08 & 0.08 & 0.07 & 0.08 & 0.07 & 0.10 & 0.06 & 0.22 \\
\hline $\mathrm{Al}_{2} \mathrm{O}_{3}$ & 12.90 & 12.11 & 12.67 & 12.67 & 10.32 & 14.34 & 12.55 & 11.50 \\
\hline $\mathrm{Fe}_{2} \mathrm{O}_{3}$ & 1.41 & 1.26 & 1.42 & 0.95 & 0.61 & 0.71 & 0.98 & 0.61 \\
\hline $\mathrm{MnO}$ & 0.02 & 0.03 & 0.00 & 0.03 & 0.03 & 0.00 & 0.02 & 0.01 \\
\hline $\mathrm{MgO}$ & nd & nd & nd & nd & nd & nd & nd & nd \\
\hline $\mathrm{CaO}$ & 0.78 & 0.69 & 0.83 & 1.18 & 0.74 & 1.70 & 1.09 & 0.71 \\
\hline $\mathrm{Na}_{2} \mathrm{O}$ & 3.69 & 3.23 & 3.30 & 3.53 & 3.14 & 3.81 & 2.87 & 2.98 \\
\hline $\mathrm{K}_{2} \mathrm{O}$ & 4.72 & 4.13 & 5.11 & 4.67 & 4.15 & 4.11 & 4.67 & 4.44 \\
\hline $\mathrm{H}_{2} \mathrm{O}$ & 0.75 & 1.58 & 4.31 & 4.09 & 1.30 & 0.46 & 3.67 & 0.75 \\
\hline $\begin{array}{c}(\mu \mathrm{g} / \mathrm{g}) \\
\mathrm{B}\end{array}$ & 28.04 & 12.86 & 34.65 & 33.70 & 24.90 & 24.00 & 35.90 & 23.70 \\
\hline $\mathrm{Cl}$ & 362.36 & 21.39 & 643.83 & 720.00 & 108.00 & 186.00 & 670.00 & 123.00 \\
\hline $\mathrm{Nd}$ & 50.87 & 50.94 & 65.88 & 49.00 & 36.00 & 58.00 & 30.00 & 31.00 \\
\hline $\mathrm{Sm}$ & 4.98 & 4.83 & 4.82 & 4.40 & 4.10 & 5.60 & 3.90 & 4.40 \\
\hline Gd & 6.51 & 6.41 & 6.29 & 5.30 & 5.30 & 6.40 & 4.80 & 5.50 \\
\hline Co & 14.7 & & 9.6 & & & & & \\
\hline $\mathrm{Ni}$ & nd & & nd & & & & & \\
\hline $\mathrm{Cr}$ & 2.0 & & nd & & & & & \\
\hline V & nd & & nd & & & & & \\
\hline $\mathrm{Pb}$ & nd & & nd & & & & & \\
\hline $\mathrm{Sr}$ & nd & & nd & & & & & \\
\hline $\mathrm{Rb}$ & 173.9 & & 166.3 & & & & & \\
\hline $\mathrm{Ba}$ & 677.8 & & 702.7 & & & & & \\
\hline $\mathrm{Zr}$ & 99.5 & & 104.4 & & & & & \\
\hline $\mathrm{Nb}$ & nd & & nd & & & & & \\
\hline Th & 16.4 & & 15.9 & & & & & \\
\hline $\mathrm{Y}$ & nd & & nd & & & & & \\
\hline $\mathrm{La}$ & 32.5 & & 30.8 & & & & & \\
\hline $\mathrm{Ce}$ & 69.5 & & 63.4 & & & & & \\
\hline $\mathrm{Pr}$ & nd & & nd & & & & & \\
\hline $\mathrm{Eu}$ & 0.4 & & 0.5 & & & & & \\
\hline $\mathrm{Gd}$ & nd & & nd & & & & & \\
\hline Dy & nd & & nd & & & & & \\
\hline Ho & 1.6 & & 1.3 & & & & & \\
\hline $\mathrm{Er}$ & nd & & nd & & & & & \\
\hline $\mathrm{Yb}$ & 3.5 & & 3.4 & & & & & \\
\hline Lu & 0.4 & & 0.1 & & & & & \\
\hline $\mathrm{Li}$ & nd & & nd & & & & & \\
\hline $\mathrm{Tb}$ & 1.0 & & 1.0 & & & & & \\
\hline $\mathrm{Tm}$ & 0.5 & & 0.5 & & & & & \\
\hline $\mathrm{Hf}$ & 4.4 & & 4.1 & & & & & \\
\hline $\mathrm{Ta}$ & 1.6 & & 1.4 & & & & & \\
\hline $\mathrm{U}$ & 4.0 & & 4.4 & & & & & \\
\hline
\end{tabular}


390 P. Kiss et al.

Table 2 cont.

\begin{tabular}{|c|c|c|c|c|c|c|c|c|}
\hline Samples & SMP-3 & SMP-5 & SMP-9 & SMP-10 & 1151 & 1173 & MKT-3 & ASU-2 \\
\hline Type & perlite & perlite & perlite & perlite & S-rhyolite & S-rhyolite & S-rhyolite & S-rhyolite \\
\hline Location & $\begin{array}{c}\text { Slansky } \\
\text { Mts } \\
\text { Brezina }\end{array}$ & $\begin{array}{c}\text { Slansky } \\
\text { Mts } \\
\text { Bysta }\end{array}$ & $\begin{array}{l}\text { Zemplin, } \\
\text { Szölöske }\end{array}$ & $\begin{array}{l}\text { Zemplin, } \\
\text { Szölöske }\end{array}$ & $\begin{array}{c}\text { Drillhole } \\
\text { Eh 13, } \\
106,0- \\
114,8 \mathrm{~m}\end{array}$ & $\begin{array}{l}\text { Abaúj- } \\
\text { szántó, } \\
\text { Sátor-hill }\end{array}$ & $\begin{array}{c}\text { Mád, } \\
\text { Király-top }\end{array}$ & $\begin{array}{c}\text { Abaúj- } \\
\text { szántó, } \\
\text { Süveges- } \\
\text { hill }\end{array}$ \\
\hline Age & nd & nd & nd & nd & $11^{* \star}$ & $11.3^{\star \star}$ & $11.7+$ & $11.11+$ \\
\hline $\begin{array}{l}\mathrm{w}+\% \\
\mathrm{SiO}_{2}\end{array}$ & 76.10 & 74.47 & 75.92 & 75.38 & 74.86 & 77.58 & 74.42 & 72.58 \\
\hline $\mathrm{TiO}_{2}$ & 0.08 & 0.41 & 0.19 & 0.19 & 0.10 & 0.08 & 0.11 & 0.26 \\
\hline $\mathrm{Al}_{2} \mathrm{O}_{3}$ & 13.52 & 15.89 & 14.76 & 13.71 & 13.43 & 12.08 & 14.13 & 14.95 \\
\hline $\mathrm{Fe}_{2} \mathrm{O}_{3}$ & 1.40 & 2.78 & 1.13 & 1.87 & 1.57 & 0.35 & 1.68 & 2.28 \\
\hline $\mathrm{MnO}$ & 0.03 & 0.03 & 0.05 & 0.06 & 0.05 & 0.00 & 0.03 & 0.02 \\
\hline $\mathrm{MgO}$ & nd & nd & nd & nd & nd & nd & nd & nd \\
\hline $\mathrm{CaO}$ & 1.24 & 1.34 & 1.04 & 1.57 & 0.97 & 0.57 & 0.75 & 1.19 \\
\hline $\mathrm{Na}_{2} \mathrm{O}$ & 3.20 & 1.68 & 2.26 & 2.62 & 3.81 & 3.78 & 3.56 & 4.29 \\
\hline $\mathrm{K}_{2} \mathrm{O}$ & 4.31 & 3.56 & 4.75 & 4.61 & 5.20 & 5.56 & 5.33 & 4.44 \\
\hline $\mathrm{H}_{2} \mathrm{O}$ & 5.35 & 10.22 & 4.95 & 4.01 & 0.27 & 0.42 & 0.95 & 1.85 \\
\hline $\begin{array}{c}(\mu \mathrm{g} / \mathrm{g}) \\
\mathrm{B}\end{array}$ & 31.70 & 26.00 & 36.30 & 32.40 & 46.77 & 51.80 & 40.12 & 33.99 \\
\hline $\mathrm{Cl}$ & 720.00 & 500.00 & 520.00 & 700.00 & 457.89 & 338.58 & 128.91 & 358.50 \\
\hline $\mathrm{Nd}$ & 39.00 & 21.00 & 32.00 & 34.00 & bd & 36.70 & 37.11 & bd \\
\hline $\mathrm{Sm}$ & 4.80 & 4.60 & 3.50 & 3.50 & 4.31 & 3.95 & 3.98 & 4.09 \\
\hline Gd & 5.70 & 6.00 & 4.30 & 4.00 & 5.27 & 4.79 & 5.08 & 4.98 \\
\hline Co & & & & & 1.0 & 0.5 & 5.2 & 9.6 \\
\hline $\mathrm{Ni}$ & & & & & 1.3 & 13.2 & nd & nd \\
\hline $\mathrm{Cr}$ & & & & & 12.4 & 3.8 & 1.6 & 2.0 \\
\hline V & & & & & 4.1 & 5.1 & nd & nd \\
\hline $\mathrm{Pb}$ & & & & & 58.9 & 73.0 & nd & nd \\
\hline $\mathrm{Sr}$ & & & & & 49.5 & 26.2 & nd & 74.4 \\
\hline $\mathrm{Rb}$ & & & & & 372.6 & 313.6 & 195.8 & 171.1 \\
\hline $\mathrm{Ba}$ & & & & & 670.4 & 554.8 & 679.4 & 612.6 \\
\hline $\mathrm{Zr}$ & & & & & 88.8 & 72.2 & 222.7 & 356.2 \\
\hline $\mathrm{Nb}$ & & & & & 18.4 & 15.2 & nd & nd \\
\hline Th & & & & & 23.6 & 17.3 & 21.9 & 17.6 \\
\hline $\mathrm{Y}$ & & & & & 32.4 & 22.8 & nd & nd \\
\hline La & & & & & 33.2 & 25.1 & 30.7 & 35.8 \\
\hline $\mathrm{Ce}$ & & & & & 91.7 & 74.9 & 57.9 & 63.2 \\
\hline $\mathrm{Pr}$ & & & & & 7.9 & 6.4 & nd & nd \\
\hline $\mathrm{Eu}$ & & & & & 0.3 & 0.2 & 0.5 & 0.9 \\
\hline $\mathrm{Gd}$ & & & & & 6.0 & 3.5 & nd & nd \\
\hline Dy & & & & & 4.8 & 4.0 & nd & nd \\
\hline $\mathrm{Ho}$ & & & & & 1.2 & 0.8 & nd & nd \\
\hline $\mathrm{Er}$ & & & & & 3.6 & 2.3 & nd & nd \\
\hline $\mathrm{Yb}$ & & & & & 4.4 & 2.6 & 2.2 & 3.2 \\
\hline Lu & & & & & 0.5 & 0.3 & 0.8 & 0.3 \\
\hline $\mathrm{Li}$ & & & & & 32.9 & 0.7 & nd & nd \\
\hline $\mathrm{Tb}$ & & & & & 0.9 & 0.5 & 0.7 & 0.8 \\
\hline $\mathrm{Tm}$ & & & & & 0.5 & 0.3 & 0.4 & 0.4 \\
\hline $\mathrm{Hf}$ & & & & & 3.3 & 2.5 & 5.1 & 8.1 \\
\hline $\mathrm{Ta}$ & & & & & 1.4 & 1.0 & 1.2 & 1.5 \\
\hline$U$ & & & & & 93.6 & 11.6 & 3.9 & 3.5 \\
\hline
\end{tabular}

calc-alkaline rocks measured with PGAA (rhyolite, abbr: N-rhyolite = northern rhyolite, S-rhyolite = southern rhyolite, al rhyolite $=$ altered rhyolite, $\mathrm{nd}=$ non determined, $\mathrm{bd}=$ below detection limit). Major elements are in wt\% and trace elements are in $\mu \mathrm{g} / \mathrm{g}$. *Balogh et al. 1983, **Pécskay et al. 1986, +this study, ++Pécskay and Molnár, 2002 
Table 2 cont.

\begin{tabular}{|c|c|c|c|c|c|c|c|c|}
\hline Samples & EVT-2 & EVT-3 & ENP-4 & ENP-1 & $\mathrm{MDH} 3$ & 485 & 891 & 1112 \\
\hline Type & S-rhyolite & S-rhyolite & S-rhyolite & S-rhyolite & al-rhyolite & $\mathrm{N}$-dacite & $\mathrm{N}$-dacite & N-dacite \\
\hline Location & $\begin{array}{c}\text { Erdő- } \\
\text { bénye, } \\
\text { Veres-top }\end{array}$ & $\begin{array}{c}\text { Erdő- } \\
\text { bénye, } \\
\text { Veres-top }\end{array}$ & $\begin{array}{c}\text { Erdő- } \\
\text { bénye, } \\
\text { Nagy } \\
\text { Páca }\end{array}$ & $\begin{array}{c}\text { Erdő- } \\
\text { bénye, } \\
\text { Nagy } \\
\text { Páca }\end{array}$ & $\begin{array}{c}\text { Mád, Diós } \\
\text { hill }\end{array}$ & $\begin{array}{c}\text { Sátoralja- } \\
\text { újhely } \\
\text { Vár-hill }\end{array}$ & $\begin{array}{c}\text { Füzér, } \\
\text { Kopaszka }\end{array}$ & $\begin{array}{l}\text { Sátoralja- } \\
\text { újhely, } \\
\text { Néma-hill }\end{array}$ \\
\hline Age & $11.75+$ & $11.01+$ & nd & nd & nd & $11.5^{*}$ & $11.8^{* *}$ & $12.4^{* *}$ \\
\hline $\begin{array}{l}\mathrm{wt} \% \\
\mathrm{SiO}_{2}\end{array}$ & 76.34 & 75.01 & 72.30 & 74.65 & 83.59 & 67.96 & 65.27 & 64.31 \\
\hline $\mathrm{TiO}_{2}$ & 0.10 & 0.11 & 0.13 & 0.10 & 1.35 & 0.58 & 0.74 & 0.98 \\
\hline $\mathrm{Al}_{2} \mathrm{O}_{3}$ & 13.17 & 13.41 & 15.16 & 13.52 & 13.10 & 15.69 & 17.53 & 16.58 \\
\hline $\mathrm{Fe}_{2} \mathrm{O}_{3}$ & 1.80 & 1.87 & 1.49 & 1.56 & 1.83 & 4.51 & 4.91 & 3.23 \\
\hline $\mathrm{MnO}$ & 0.00 & 0.03 & 0.02 & 0.02 & bd & 0.05 & 0.08 & 0.16 \\
\hline $\mathrm{MgO}$ & nd & nd & nd & nd & nd & 1.20 & nd & 1.31 \\
\hline $\mathrm{CaO}$ & 0.00 & 0.89 & 0.97 & 1.17 & 0.14 & 3.74 & 5.06 & 7.57 \\
\hline $\mathrm{Na}_{2} \mathrm{O}$ & 3.69 & 3.50 & 4.52 & 4.24 & bd & 3.14 & 3.62 & 3.41 \\
\hline $\mathrm{K}_{2} \mathrm{O}$ & 4.90 & 5.17 & 5.42 & 4.74 & $\mathrm{bd}$ & 3.11 & 2.78 & 2.44 \\
\hline $\mathrm{H}_{2} \mathrm{O}$ & 0.81 & 2.65 & 0.46 & 0.42 & 5.37 & 2.17 & 1.26 & 0.95 \\
\hline $\begin{array}{c}(\mu g / g) \\
B\end{array}$ & 46.56 & 52.12 & 45.60 & 47.30 & 1.24 & 28.73 & 20.06 & 10.16 \\
\hline $\mathrm{Cl}$ & 208.13 & 609.03 & 550.00 & 213.00 & bd & 123.23 & 81.04 & 43.87 \\
\hline $\mathrm{Nd}$ & 46.30 & 46.95 & 42.00 & 44.00 & 58.91 & 28.74 & bd & 26.04 \\
\hline $\mathrm{Sm}$ & 4.08 & 4.01 & 4.20 & 4.80 & 5.42 & 3.14 & 3.87 & 3.92 \\
\hline $\mathrm{Gd}$ & 5.42 & 4.75 & 4.80 & 5.70 & 6.08 & 3.96 & 5.36 & 5.18 \\
\hline Co & 7.4 & 7.4 & & & & 10.3 & 13.1 & 15.1 \\
\hline $\mathrm{Ni}$ & nd & nd & & & & 7.0 & 5.2 & 12.2 \\
\hline $\mathrm{Cr}$ & 2.1 & 2.7 & & & & 49.2 & 18.1 & 59.4 \\
\hline V & nd & nd & & & & 86.5 & 88.7 & 137.4 \\
\hline $\mathrm{Pb}$ & nd & nd & & & & 58.4 & 27.4 & 24.9 \\
\hline $\mathrm{Sr}$ & 58.7 & 87.8 & & & & 119.9 & 183.3 & 122.4 \\
\hline $\mathrm{Rb}$ & 175.1 & 213.2 & & & & 155.7 & 161.9 & 128.3 \\
\hline $\mathrm{Ba}$ & 589.7 & 627.0 & & & & 556.4 & 682.5 & 370.0 \\
\hline $\mathrm{Zr}$ & 188.2 & 187.6 & & & & 127.4 & 96.3 & 119.9 \\
\hline $\mathrm{Nb}$ & nd & nd & & & & 11.5 & 11.3 & 11.6 \\
\hline Th & 21.5 & 22.1 & & & & 14.2 & 8.6 & 12.2 \\
\hline $\mathrm{Y}$ & nd & nd & & & & 26.6 & 33.3 & 27.6 \\
\hline La & 34.4 & 35.3 & & & & 32.4 & 34.6 & 26.7 \\
\hline $\mathrm{Ce}$ & 69.7 & 67.6 & & & & 63.3 & 85.0 & 62.0 \\
\hline $\mathrm{Pr}$ & nd & nd & & & & 7.3 & 9.5 & 7.2 \\
\hline $\mathrm{Eu}$ & 0.9 & 0.9 & & & & 0.9 & 0.9 & 0.8 \\
\hline $\mathrm{Gd}$ & nd & nd & & & & 5.0 & 6.4 & 4.8 \\
\hline Dy & nd & nd & & & & 4.8 & 6.3 & 4.9 \\
\hline $\mathrm{Ho}$ & nd & 1.2 & & & & 1.1 & 1.2 & 1.0 \\
\hline $\mathrm{Er}$ & nd & nd & & & & 3.1 & 3.7 & 3.2 \\
\hline $\mathrm{Yb}$ & 2.8 & 2.5 & & & & 3.2 & 3.0 & 3.1 \\
\hline $\mathrm{Lu}$ & 0.4 & 0.1 & & & & 0.5 & 0.4 & 0.4 \\
\hline $\mathrm{Li}$ & nd & nd & & & & 28.3 & $\mathrm{bd}$ & $\mathrm{bd}$ \\
\hline $\mathrm{Tb}$ & 0.7 & 0.7 & & & & 0.8 & 0.9 & 0.7 \\
\hline $\mathrm{Tm}$ & 0.4 & 0.5 & & & & 0.5 & 0.6 & 0.5 \\
\hline $\mathrm{Hf}$ & 4.9 & 4.9 & & & & 4.2 & 3.1 & 3.8 \\
\hline $\mathrm{Ta}$ & 1.4 & 1.3 & & & & 1.0 & 0.8 & 1.0 \\
\hline $\mathrm{U}$ & 5.9 & 5.3 & & & & 4.6 & 7.6 & 6.2 \\
\hline
\end{tabular}


Table 2 cont.

\begin{tabular}{|c|c|c|c|c|c|c|c|c|}
\hline Samples & 1206 & GOK-1 & SMP-4 & 543 & 715 & 880 & EIK-3 & SZP-2 \\
\hline Type & N-dacite & N-dacite & N-dacite & S-dacite & S-dacite & S-dacite & S-dacite & S-dacite \\
\hline Location & \begin{tabular}{|c|} 
Vágás- \\
huta, \\
Fekete-hill
\end{tabular} & $\begin{array}{l}\text { Gönc, } \\
\text { outcrop }\end{array}$ & $\begin{array}{c}\text { Slansky } \\
\text { Mts. }\end{array}$ & $\begin{array}{c}\text { Tarcal, } \\
\text { Kopasz- } \\
\text { hill }\end{array}$ & $\begin{array}{l}\text { Tokaj, } \\
\text { Patkó }\end{array}$ & $\begin{array}{l}\text { Bodrog- } \\
\text { szegi, } \\
\text { Cserepes }\end{array}$ & $\begin{array}{l}\text { Erdőbé- } \\
\text { nye, Iván- } \\
\text { stone }\end{array}$ & $\begin{array}{l}\text { Szegi, } \\
\text { Poklos }\end{array}$ \\
\hline Age & $12.9^{\star *}$ & $12.12^{+}$ & nd & $10.3^{*}$ & $10.5^{*}$ & $10.1^{* *}$ & $10.57^{+}$ & $10.1^{+}$ \\
\hline $\begin{array}{l}(w t \%) \\
\mathrm{SiO}_{2}\end{array}$ & 64.17 & 64.31 & 69.41 & 64.70 & 67.26 & 63.35 & 69.08 & 65.11 \\
\hline $\mathrm{TiO}_{2}$ & 0.71 & 0.67 & 0.21 & 0.76 & 0.82 & 0.99 & 0.40 & 1.02 \\
\hline $\mathrm{Al}_{2} \mathrm{O}_{3}$ & 15.57 & 18.24 & 17.30 & 15.44 & 15.41 & 15.98 & 15.78 & 18.21 \\
\hline $\mathrm{Fe}_{2} \mathrm{O}_{3}$ & 5.95 & 4.79 & 3.31 & 5.60 & 5.25 & 6.58 & 3.64 & 3.15 \\
\hline $\mathrm{MnO}$ & 0.12 & 0.09 & 0.06 & 0.11 & 0.10 & 0.18 & 0.08 & 0.02 \\
\hline $\mathrm{MgO}$ & 2.66 & nd & nd & 2.21 & nd & 1.27 & nd & nd \\
\hline $\mathrm{CaO}$ & 5.11 & 6.70 & 3.21 & 4.23 & 3.74 & 4.21 & 2.51 & 4.15 \\
\hline $\mathrm{Na}_{2} \mathrm{O}$ & 3.01 & 2.74 & 3.42 & 3.57 & 3.72 & 3.49 & 4.07 & 4.88 \\
\hline $\mathrm{K}_{2} \mathrm{O}$ & 2.69 & 2.47 & 2.90 & 3.37 & 3.69 & 3.95 & 4.43 & 3.46 \\
\hline $\mathrm{H}_{2} \mathrm{O}$ & 1.13 & 1.39 & 3.85 & 1.44 & 1.29 & 2.02 & 2.25 & 1.22 \\
\hline $\begin{array}{c}(\mu \mathrm{g} / \mathrm{g}) \\
\mathrm{B}\end{array}$ & 21.03 & 24.08 & 14.60 & 19.66 & 22.20 & 38.16 & 38.64 & 17.96 \\
\hline $\mathrm{Cl}$ & 192.36 & 58.86 & 27.00 & 42.24 & 53.29 & 524.50 & 460.12 & 72.69 \\
\hline $\mathrm{Nd}$ & 31.02 & 33.14 & 29.00 & 31.33 & 33.88 & 50.26 & 46.30 & 64.95 \\
\hline $\mathrm{Sm}$ & 3.29 & 3.36 & 3.70 & 3.81 & 4.01 & 5.85 & 4.35 & 7.97 \\
\hline $\mathrm{Gd}$ & 4.30 & 4.33 & 4.70 & 4.69 & 4.97 & 7.95 & 5.45 & 11.23 \\
\hline Co & & 12.8 & & 13.7 & 11.7 & 11.7 & 10.1 & 4.6 \\
\hline $\mathrm{Ni}$ & & nd & & 19.2 & 51.3 & 5.0 & nd & $\mathrm{nd}$ \\
\hline $\mathrm{Cr}$ & & 54.9 & & 46.7 & 27.7 & 40.0 & 4.9 & 29.9 \\
\hline $\mathrm{V}$ & & nd & & 90.3 & 80.8 & 50.5 & nd & nd \\
\hline $\mathrm{Pb}$ & & nd & & 38.6 & 48.4 & 35.2 & nd & nd \\
\hline $\mathrm{Sr}$ & & 220.2 & & 185.2 & 176.4 & 190.0 & 185.5 & 299.8 \\
\hline $\mathrm{Rb}$ & & 80.1 & & 206.0 & 238.9 & 254.4 & 176.0 & nd \\
\hline $\mathrm{Ba}$ & & 367.4 & & 677.8 & 686.6 & 833.5 & 644.3 & 663.8 \\
\hline $\mathrm{Zr}$ & & 114.3 & & 216.1 & 250.5 & 225.9 & 438.7 & nd \\
\hline $\mathrm{Nb}$ & & nd & & 25.2 & 31.6 & 18.4 & nd & nd \\
\hline Th & & 8.9 & & 20.9 & 19.2 & 12.4 & 16.5 & 10.9 \\
\hline$Y$ & & nd & & 31.8 & 41.8 & 45.8 & nd & nd \\
\hline $\mathrm{La}$ & & 25.0 & & 48.3 & 47.3 & 45.6 & 38.9 & 27.2 \\
\hline $\mathrm{Ce}$ & & 49.2 & & 105.5 & 130.7 & 101.7 & 72.9 & 57.8 \\
\hline $\mathrm{Pr}$ & & nd & & 11.9 & 13.0 & 11.6 & nd & nd \\
\hline $\mathrm{Eu}$ & & 1.0 & & 1.2 & 1.0 & 1.3 & 1.0 & 1.2 \\
\hline $\mathrm{Gd}$ & & nd & & 5.7 & 6.7 & 8.0 & nd & nd \\
\hline Dy & & nd & & 5.6 & 6.8 & 8.6 & nd & nd \\
\hline $\mathrm{Ho}$ & & nd & & 1.1 & 1.2 & 1.7 & nd & 2.7 \\
\hline $\mathrm{Er}$ & & nd & & 3.4 & 3.6 & 5.1 & nd & nd \\
\hline $\mathrm{Yb}$ & & 2.4 & & 3.3 & 3.9 & 5.5 & 2.9 & 1.9 \\
\hline $\mathrm{Lu}$ & & 0.1 & & 0.6 & 0.4 & 0.7 & 0.2 & 0.1 \\
\hline $\mathrm{Li}$ & & nd & & 13.8 & 47.6 & bd & nd & nd \\
\hline $\mathrm{Tb}$ & & 0.6 & & 0.9 & 1.0 & 1.4 & 0.8 & 0.7 \\
\hline $\mathrm{Tm}$ & & 0.4 & & 0.5 & 0.6 & 0.8 & 0.4 & 0.5 \\
\hline $\mathrm{Hf}$ & & 4.0 & & 6.5 & 5.7 & 6.3 & 9.0 & 5.9 \\
\hline $\mathrm{Ta}$ & & 0.6 & & 1.7 & 2.1 & 1.0 & 1.2 & 1.1 \\
\hline $\mathrm{U}$ & & 2.0 & & 62.6 & 45.6 & 44.7 & 4.2 & 2.3 \\
\hline
\end{tabular}


Table 2 cont.

\begin{tabular}{|c|c|c|c|c|c|c|c|c|}
\hline Samples & 484 & 1116 & 1149 & 1201 & 4024 & 4244 & 5171 & HMA-1 \\
\hline Type & basalt & andesite & adesite & andesite & andesite & andesite & andesite & andesite \\
\hline Location & $\begin{array}{c}\text { Sáros- } \\
\text { patak-10, } \\
\text { drillhole } \\
\text { No. } 10\end{array}$ & $\begin{array}{c}\text { Füzér, } \\
\text { Vár-hegy }\end{array}$ & $\begin{array}{c}\mathrm{F}-2 \\
\text { drillhole, } \\
143.7- \\
146,7 \mathrm{~m}\end{array}$ & $\begin{array}{l}\text { Füzér, } \\
\text { Nagy- } \\
\text { Milic }\end{array}$ & $\begin{array}{l}\text { Nagyhuta, } \\
\text { Mlaka- } \\
\text { meadow }\end{array}$ & Regéc & $\begin{array}{c}\text { Telki- } \\
\text { bánya, } \\
\text { Nagy } \\
\text { Sertés-hill }\end{array}$ & $\begin{array}{c}\text { Holló- } \\
\text { háza, } \\
\text { Magos-hill }\end{array}$ \\
\hline Age & $9.4^{*}$ & $10.9^{* \star}$ & $12.9^{\star \star}$ & $10.9^{\star *}$ & $12.25^{+}$ & $12.97^{++}$ & $11.67^{+}$ & nd \\
\hline $\begin{array}{l}(\mathrm{wt} \%) \\
\mathrm{SiO}_{2}\end{array}$ & 50.12 & 61.43 & 62.22 & 58.95 & 62.04 & 61.22 & 59.91 & 61.06 \\
\hline $\mathrm{TiO}_{2}$ & 1.13 & 0.87 & 0.81 & 0.94 & 0.74 & 0.67 & 0.84 & 0.55 \\
\hline $\mathrm{Al}_{2} \mathrm{O}_{3}$ & 15.58 & 17.15 & 15.60 & 17.98 & 14.73 & 15.86 & 13.74 & 16.18 \\
\hline $\mathrm{Fe}_{2} \mathrm{O}_{3}$ & 8.41 & 6.64 & 6.32 & 7.42 & 7.12 & 6.58 & 7.82 & 5.80 \\
\hline $\mathrm{MnO}$ & 0.16 & 0.14 & 0.11 & 0.15 & 0.14 & 0.12 & 0.14 & 0.10 \\
\hline $\mathrm{MgO}$ & 12.44 & 2.41 & 3.84 & 3.28 & 4.59 & 4.24 & 7.50 & 5.09 \\
\hline $\mathrm{CaO}$ & 8.15 & 5.60 & 5.58 & 6.48 & 5.76 & 6.53 & 5.65 & 6.41 \\
\hline $\mathrm{Na}_{2} \mathrm{O}$ & 2.56 & 3.49 & 3.42 & 3.12 & 2.52 & 2.44 & 2.30 & 3.21 \\
\hline $\mathrm{K}_{2} \mathrm{O}$ & 1.45 & 2.28 & 2.10 & 1.67 & 2.35 & 2.32 & 2.10 & 1.60 \\
\hline $\mathrm{H}_{2} \mathrm{O}$ & 1.84 & 1.49 & 0.65 & 1.11 & 1.38 & 2.26 & 1.19 & 1.98 \\
\hline $\begin{array}{c}(\mu \mathrm{g} / \mathrm{g}) \\
\mathrm{B}\end{array}$ & 10.34 & 12.35 & 10.06 & 16.08 & 13.22 & 14.25 & 17.26 & 10.40 \\
\hline $\mathrm{Cl}$ & 13.91 & 46.46 & 45.59 & 491.21 & 35.19 & 33.10 & 30.46 & 37.00 \\
\hline $\mathrm{Nd}$ & bd & $\mathrm{bd}$ & 26.49 & 29.11 & 28.17 & bd & $\mathrm{bd}$ & 31.00 \\
\hline Sm & 2.85 & 3.41 & 2.95 & 3.18 & 3.25 & 3.27 & 3.15 & 2.49 \\
\hline Gd & 3.73 & 4.75 & 3.99 & 4.49 & 4.28 & 4.19 & 4.14 & 3.00 \\
\hline Co & & 12.7 & 17.7 & & & & & \\
\hline $\mathrm{Ni}$ & & 3.1 & 42.2 & & & & & \\
\hline $\mathrm{Cr}$ & & 14.4 & 138.2 & & & & & \\
\hline V & & 62.3 & 141.5 & & & & & \\
\hline $\mathrm{Pb}$ & & 19.8 & 23.2 & & & & & \\
\hline $\mathrm{Sr}$ & & 202.9 & 160.2 & & & & & \\
\hline $\mathrm{Rb}$ & & 107.5 & 159.5 & & & & & \\
\hline $\mathrm{Ba}$ & & 391.8 & 479.4 & & & & & \\
\hline $\mathrm{Zr}$ & & 107.5 & 192.1 & & & & & \\
\hline $\mathrm{Nb}$ & & 7.3 & 12.8 & & & & & \\
\hline Th & & 8.5 & 13.2 & & & & & \\
\hline $\mathrm{Y}$ & & 26.9 & 31.8 & & & & & \\
\hline $\mathrm{La}$ & & 25.5 & 28.9 & & & & & \\
\hline $\mathrm{Ce}$ & & 77.7 & 69.5 & & & & & \\
\hline $\mathrm{Pr}$ & & 6.7 & 7.7 & & & & & \\
\hline $\mathrm{Eu}$ & & 0.9 & 0.9 & & & & & \\
\hline $\mathrm{Gd}$ & & 4.7 & 5.3 & & & & & \\
\hline Dy & & 4.6 & 5.4 & & & & & \\
\hline $\mathrm{Ho}$ & & 1.0 & 1.1 & & & & & \\
\hline Er & & 2.6 & 3.3 & & & & & \\
\hline $\mathrm{Yb}$ & & 2.6 & 3.6 & & & & & \\
\hline Lu & & 0.4 & 0.5 & & & & & \\
\hline $\mathrm{Li}$ & & $\mathrm{bd}$ & 52.2 & & & & & \\
\hline $\mathrm{Tb}$ & & 0.7 & 0.9 & & & & & \\
\hline $\mathrm{Tm}$ & & 0.5 & 0.5 & & & & & \\
\hline $\mathrm{Hf}$ & & 2.7 & 5.7 & & & & & \\
\hline $\mathrm{Ta}$ & & 0.7 & 1.0 & & & & & \\
\hline $\mathrm{U}$ & & 1.5 & 10.9 & & & & & \\
\hline
\end{tabular}



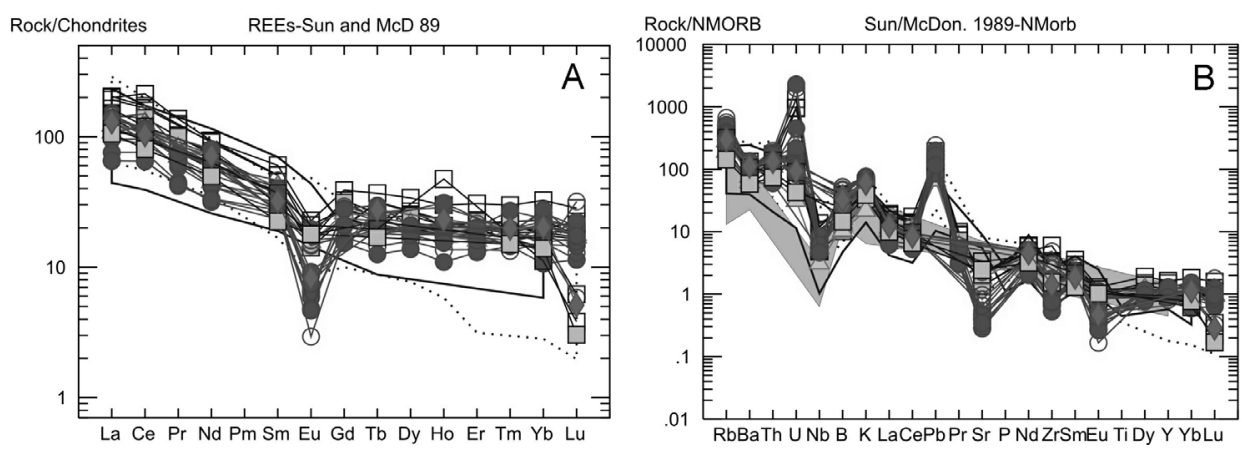

Fig. 4

Multi element diagrams normalised for A) chondrites (REE-diagram) and B) NMORB values (both reference values are from Sun and McDonough 1989). The symbols are the same as in Fig. 3

Th. Another important characteristic of the volcanic rocks from the Tokaj Mts is the high variation of $\mathrm{Zr}$ concentrations (between 39 and $406 \mu \mathrm{g} / \mathrm{g}$ ). Note that the most enriched samples in $\mathrm{Zr}$ are the dacite samples from the southern part of the Tokaj Mts. One interesting feature present in the case of the Tokaj Mts samples is the characteristic $U$ anomaly, which is not visible in other areas where a similar geotectonic setting is obvious.

The other trace elements used for discrimination do not show any unusual characteristic compared to other subduction-related volcanic rocks; however some obvious trends or differences are present. These are the following: 1) the Ba and $\mathrm{Th}$ contents display weak positive correlations with the $\mathrm{SiO}_{2}$ content of rocks (Fig. $3 \mathrm{H}, \mathrm{J}$ ). In the case of Th the southern rhyolite samples of the Tokaj Mts contain slightly more Th (discounting two samples where the concentration varies between 16 and $35 \mu \mathrm{g} / \mathrm{g}$ ) than the northern ones (between 6 and $21 \mu / \mathrm{g}$ ); this is in good agreement with the distribution of airborne Th-anomalies. In contrast $\mathrm{Sr}$ shows a negative correlation with the $\mathrm{SiO}_{2}$ (Fig. 3F), and also with the $\mathrm{K}_{2} \mathrm{O}$ content of rocks, while in the case of $\mathrm{Rb}$ no clear correlation could be established.

$\mathrm{K} /$ Ar ages

The K/Ar ages of samples studied range from 13.4 to 9.4 Ma and show some differences in their geochemical characteristics as a function of their age. The age vs. $\mathrm{SiO}_{2}$ diagram (Fig. 5A) shows a significant difference between the northern and southern rocks. In the northern part of the Tokaj Mts the rocks with different $\mathrm{SiO}_{2}$ contents were contemporaneously generated between 13.4 and $10.9 \mathrm{Ma}$, while in the southern Tokaj Mts only andesite and rhyolite were formed in this time interval; the dacite is much younger (10.4-10.1 Ma).

La also shows a trend with the K/Ar age: it increases with decreasing age of the samples. 

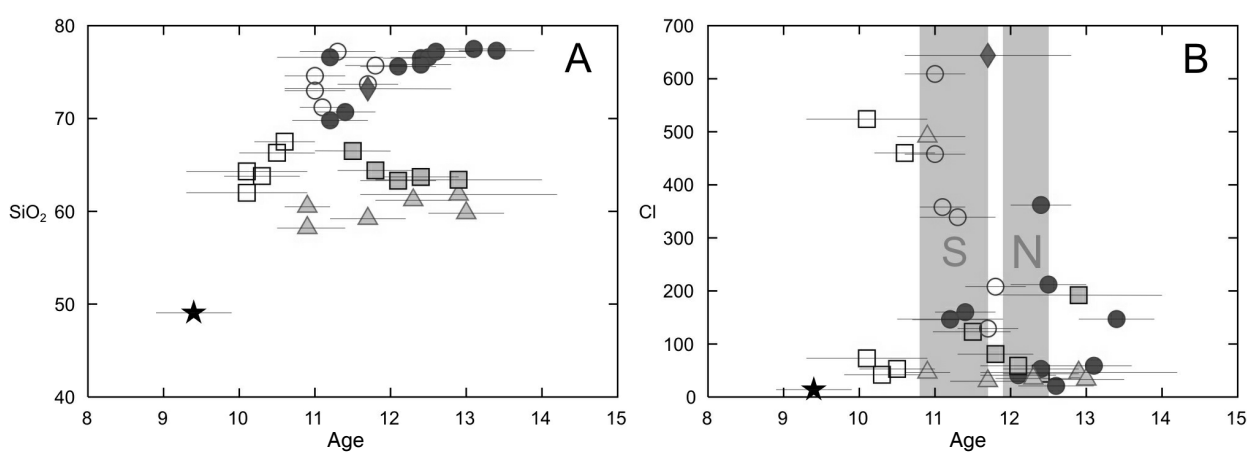

Fig. 5

$\mathrm{K} /$ Ar ages (Ma) plotted against the $\mathrm{SiO}_{2}$ content of rocks (A). K/Ar ages vs $\mathrm{Cl}$ content (B) with the age of hydrothermal activity (12.5-11.9 Ma on the northern part and 11.7-10.8 Ma on the south (Pécskay and Molnár 2002) represented by the shaded intervals. The symbols are the same as in Fig. 3. $\mathrm{N}=$ the age of hydrothermal activity for the northern part of the mountains, $S=$ the age hydrothermal activity for the southern Tokaj Mts

Rhyolite in the southern Tokaj Mts is coeval with the hydrothermal activity (Fig. 5B). The northern rhyolite fields also provided some samples with K-Ar ages identical to the hydrothermal activity, but there is also older and younger rhyolite. Rhyolite samples from volcanic centers in the northern part of the Tokaj Mts are spatially unrelated to mineralized zones. It is also remarkable that rhyolite, being coeval with hydrothermal activity in the southern part of the Tokaj Mts, contains up to $0.6 \mathrm{wt} \%$ chlorine, whereas the northern rhyolite - with exception of perlite - has less than $0.4 \mathrm{wt} \% \mathrm{Cl}$-contents (Table 1 and Fig. 5B).

\section{Discussion}

In this section we try to analyze the process(es) responsible for magma generation in the Tokaj Mts and to compare them with similar volcanic fields from the Carpathian-Pannonian Region (Harangi et al. 2007), from the Aeolian Islands (Tonarini et al. 2004) as well as from the Kuriles (Ishikawa and Tera 1997). Additionally we aimed at finding some correlation between the presence or absence of the traces of ore generation and changes of the geochemical characteristics of the mainly acidic volcanic rocks.

Boron is a very good tracer of the upper mantle processes during subduction and the range of boron concentrations found in volcanic rocks of the Tokaj Mts (Table 1) is typical for subduction-related volcanic settings (Leeman et al. 1994). The decrease of B concentrations in volcanic rocks toward the back-arc regions is a well-known phenomenon in those settings (Ryan et al. 1995). Based on Ishikawa and Tera (1997), we also attempted to plot B as well as K/B*10-3 values of samples against the relative distances of their localities from the subduction suture zone. This value is a good tracer of the changes of the fluid composition behind the volcanic front because mobilization of $\mathrm{K}$ from the subducting material 

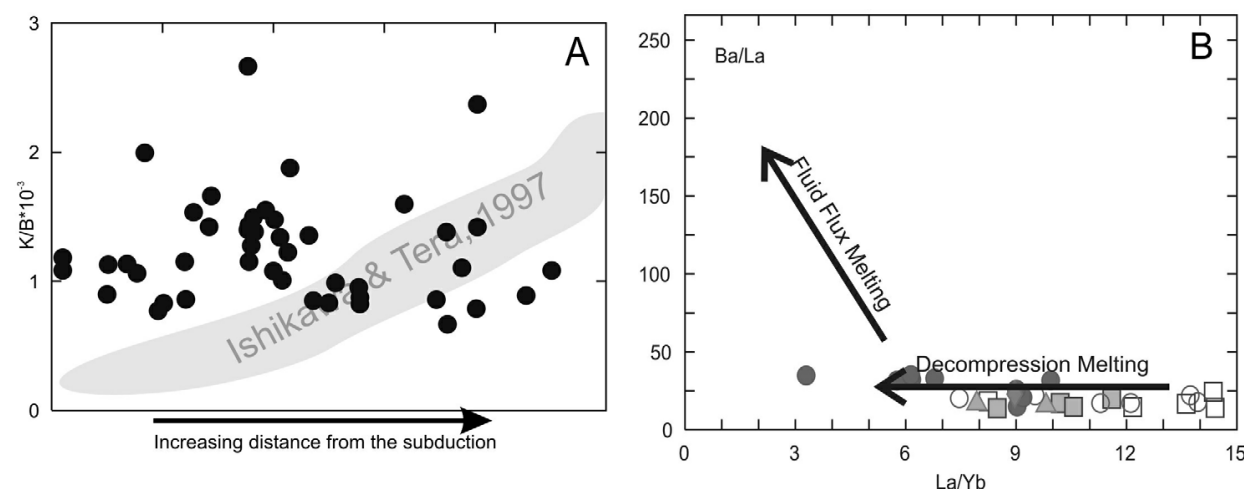

Fig. 6

A) $\mathrm{B} / \mathrm{K}^{*} 10^{3}$ plotted against increasing distance from subduction suture zone. Because we used relative distance, the $\mathrm{X}$-axis represents increasing distance from the trench without values. Note that all samples plotted as black dottes. B) $\mathrm{Ba} / \mathrm{La}$ vs $\mathrm{La} / \mathrm{Yb}$ diagram used to make difference between decompression and flux melting processes and to determine the producer of the melting in the Tokaj Mts. For explanation of the symbols see Fig. 3

requires higher temperature. Unlike potassium, large amounts of boron are released at the location of the volcanic front, so its concentration decreases in the direction of the back-arc basin, with a parallel increase in the K/B ratio, some tens of $\mathrm{km}$ behind the volcanic front. However, we do not know the accurate location of the subduction zone during the generation of magmatism; therefore we established a most probable hypothetical subduction front, which is subparallel to the Szamos line (= Szamos Fault in Fig. 1). Figure 6A shows that the K/B ${ }^{*} 10-3$ values do not show any correlation with the distance from our hypothetical trench. We also chose other hypothetical subduction front settings with variable relative distances of the samples by choosing various samples as the basis of the comparison. The results are the same: the boron contents of the rocks from the Tokaj Mts do not show systematic spatial variation. Therefore we can rule out the direct influence of subduction-related components in the generation of magmas.

On the basis of our geochemical data we were able to infer the magma generation processes in the Tokaj Mts during Late Miocene times. On the basis of the $\mathrm{Ba} / \mathrm{La}$ vs. $\mathrm{La} / \mathrm{Yb}$ diagram (published by Cameron et al. 2003; Fig. 6) we can determine the parent melt of the magmas in the Tokaj Mts. This diagram is useful for separating lava with significant slab fluid influx from that generated by decompressional melting. It is important to note that a high degree of LREE mobilization from a subducted slab would reduce the effectiveness of $\mathrm{La} / \mathrm{Yb}$ as a measure of the degree of mantle melting (Cameron et al. 2003). The low Ba/La ratios in the Tokaj Mts lavas allows us to interpret that the parent melt of the studied rock samples was generated by decompression melting. On the other hand, it can also be seen that most of the northern rhyolite contains much lower amounts of $\mathrm{La} / \mathrm{Yb}$ than the other rocks. The samples from the Tokaj Mts (as well as the Aeolian samples) exhibit a horizontal trend. The absence of any 

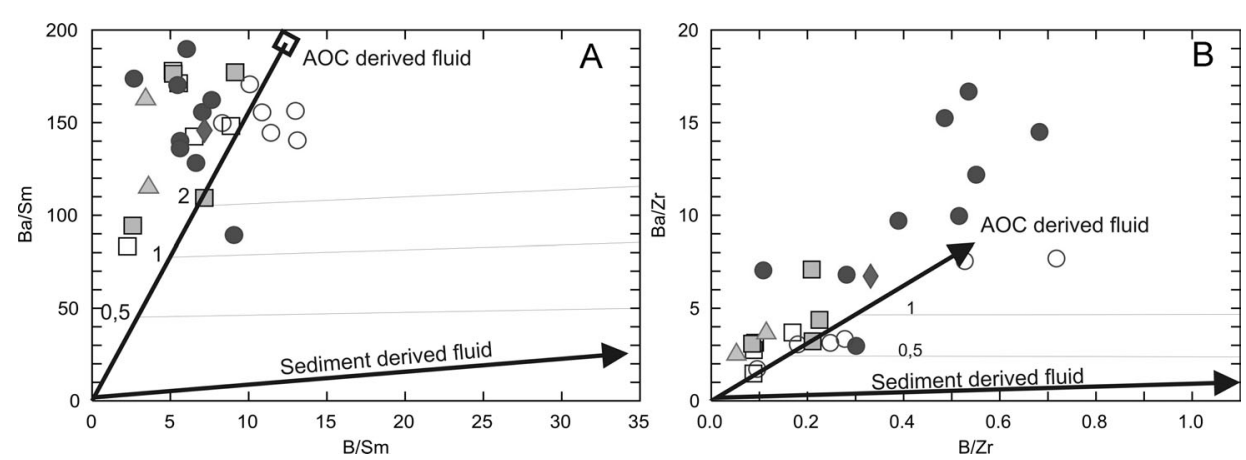

Fig. 7

Comparative diagrams of the B and Ba concentrations in the rocks as a function of either Sm (A) and $\mathrm{Zr}$ (B) concentrations to determine the origin of the fluid metasomatised the mantle(wedge) above the subtucting slab. The symbols are the same as in Fig. 3

relationship between systematic slab input and degree of melting also suggests a more prominent role of decompression melting beneath the Tokaj Mts. The difference in the $\mathrm{La} / \mathrm{Yb}$ values of rocks are thought to be due to the different degree of mantle melting (Cameron et al. 2003) as suggested by Fig. 6B; however, one cannot rule out the affect of fractional crystallization or assimilation of some crustal melts on these values (Davidson et al. 2007; Moyen 2009).

Although decompression melting is obvious in the Tokaj Mts, the geochemical characteristics of the rocks show real subduction-related patterns, which requires some fluid addition from the subducting slab to the source region of the magmas. This explanation is refined by the $\mathrm{B} / \mathrm{Sm}$ vs. $\mathrm{Ba} / \mathrm{Sm}$ and $\mathrm{B} / \mathrm{Zr}$ vs. $\mathrm{Ba} / \mathrm{Zr}$ variation diagrams (after Sano et al. 2001; Fig. 7A, B), because $\mathrm{Zr}$ and Sm have different fluid mobility during dehydrating processes in subduction zones, where $\mathrm{Zr}$ is the most immobile element (Sano et al. 2001). On these diagrams (Fig. 7A, B) all of our samples fall near to the Altered Oceanic Crust (AOC)-derived fluid composition. This suggests similar fluid influx contributing to the generation of the magmas. The only difference present in the parental magmas is in the amount of fluid added to the mantle during fluid metasomatism. According to Kovács et al. (2004, 2007) it is hardly possible that an earlier subduction during the Paleogene-Early Miocene (namely the Budva-Pindos subduction) could metasomatize the lithospheric mantle below the Tokaj Mts. The above-mentioned difference to the addition of metasomatizing fluid to the source region of the Tokaj Mts lavas is clearly seen on the $\mathrm{B} / \mathrm{Zr}$ vs. $\mathrm{Ba} / \mathrm{Zr}$ diagram.

The $\mathrm{Rb} / \mathrm{Zr}$ vs. $\mathrm{K}_{2} \mathrm{O} / \mathrm{Na}_{2} \mathrm{O}$ diagram (Fig. $8 \mathrm{~A}$ ) also suggests that the high $\mathrm{Rb} / \mathrm{Zr}$ ratios at nearly constant $\mathrm{K}_{2} \mathrm{O} / \mathrm{Na}_{2} \mathrm{O}$ ratios are the result of a different amount of fluid added from the AOC in the Tokaj Mts. The studied samples fall on the assimilation-fractionation crystallization (AFC) trend on this diagram. This approach was also used by Seghedi et al. (2004), who stated that assimilation of some crustal melts influenced the composition of the volcanic rocks in some 

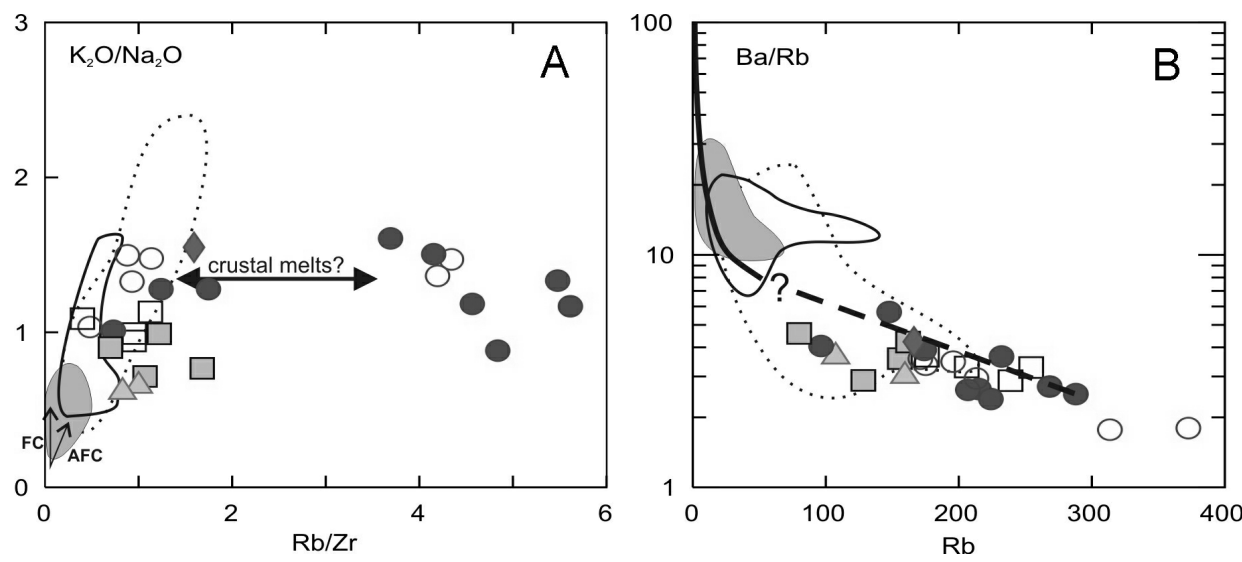

Fig. 8

$\mathrm{Rb} / \mathrm{Zr}$ vs $\mathrm{K}_{2} \mathrm{O} / \mathrm{Na}_{2} \mathrm{O}$ diagram for distinguishing crustal sources from AFC processes in the Tokaj Mts (A) and $\mathrm{Rb}$ vs $\log (\mathrm{Ba} / \mathrm{Rb})$ diagram to observe the effects of crustal melts on the Tokaj Mts lavas (B). The solid curve on "B" represents the trend of the crustal melt addition to the mantle derived basalts in NE Japan. In figure B the discursive line is the same trend for the intermediate-acidic Tokaj Mts Samples. (symbols are in Fig. 3)

other parts of the volcanic belt. Therefore we could not rule out some crustal involvement of the magmas, which is also supported by the $\mathrm{Rb}$ vs. $\log \mathrm{Ba} / \mathrm{Rb}$ diagram (Fig. 8B). Kimura and Yoshida (2006) also used the $\mathrm{Rb}$ vs. $\log \mathrm{Ba} / \mathrm{Rb}$ diagram to determine the crustal influence on compositions of Quaternary basaltic lavas in NE Japan. They stated that neither fractional crystallization nor crustal melting significantly changes the $\mathrm{Ba} / \mathrm{Rb}$ ratios, but mixing between mantle-derived magma and various crustal melts may significantly influence them. Therefore, the AFC trend and the addition of some crustal melt is highly conceivable for the parent melts of the volcanic rocks in the Tokaj Mts.

Despite the likelihood of the assimilation-fractional crystallization trend of the Tokaj Mts. Magmas, our suggestion - based on the analysis of some incompatible elements - about the origin of the melts in the Tokaj Mts is still plausible. The concentration of boron in the rock samples varies between 10.1 and $52.12 \mu \mathrm{g} / \mathrm{g}$. These values are obviously higher than the average concentration of this element in the continental crust, which is $\sim 10 \mu \mathrm{g} / \mathrm{g}(<5 \mu \mathrm{g} / \mathrm{g}$ for the lower crust, $\sim 15 \mu \mathrm{g} / \mathrm{g}$ for the upper crust; Leeman and Sisson 1996), and converge with the values of the altered oceanic crust and the deep sea sediments $(\sim 120 \mu \mathrm{g} / \mathrm{g}$ and $\sim 135 \mu \mathrm{g} / \mathrm{g}$; Leeman and Sisson 1996). Additionally boron is insensitive to fractional crystallization. Since the concentration of the other fluid mobile and incompatible elements - used in this study for determining the possible origin of the magma - correlate positively with the boron content we can state that these elements also derived from the subducting slab, therefore the conclusions of our study are relevant.

Tonarini et al. $(2001,2004)$ calculated $\mathrm{B} / \mathrm{La}$ and $\mathrm{B} / \mathrm{Nb}$ ratios for central and southern Italian calc-alkaline volcanic rocks, which have $\mathrm{B} / \mathrm{La}$ and $\mathrm{B} / \mathrm{Nb}$ ratios 
similar to those of the Tokaj Mts. The melt generation suggested for the Aeolians by Peccerillo (2005) appears to be also applicable in our case, namely: the subduction-related fluid-modified mantle melted via decompression melting. In Late Miocene times, subduction and roll-back of the subducted slab terminated east of the Tokaj Mts (Csontos et al. 1992). On the other hand, Kovács et al. (2007) revealed that extension and normal faulting played an important role in melting - of an earlier metasomatized lithospheric mantle - and rising of the magma in the Paleogene-Early Miocene in the Alpine-Carpathian-Pannonian-Dinaric region. Thus, we think that the magmas in the Tokaj Mts were formed above a metasomatized lithospheric mantle. This metasomatization could be the same described in Kovács et al. (2007) and later the magma generation occurred in a geodynamic setting very similar to that of the central and the southern Italian volcanic fields.

Another important effect which affected the magmas in the Tokaj Mts was the fractional crystallization of plagioclase, as indicated by the Eu-anomalies (Fig. 4). In the case of $\mathrm{Sr}$, which geochemically behaves similarly to Eu, the concentrations are lower than the average upper continental crust value (UCC: 350 ppm; Taylor and McLeanan 1985). This fact also appears to support assimilation of crustal melts and process of fractional crystallization because Sr concentrations are much lower in the continental crust than in mantle-derived magmas (Taylor 1980). Additionally, the variation of the La content and to a greater extent the $\mathrm{La} / \mathrm{Yb}$ ratios may be due to the addition of some crustal melts in silicic magmas (Moyen 2009 ) or due to the fractional crystallization process (Davidson et al. 2007).

The relatively high $U$ concentrations (causing the positive anomalies on the MORB-normalized multi-element diagram, Fig. 4B) can be explained by the slab influx metasomatizing the mantle area below the Tokaj Mts. The fluid mobility of $\mathrm{U}$ is similar to $\mathrm{Pb}$, which element also shows positive anomalies on our spider diagrams (Fig. 4B). To explain this phenomena, uranium is also enriched by the action of $\mathrm{H}_{2} \mathrm{O}$-rich fluids derived from the dehydration of minerals in the subducting slab (Bailey and Ragnarsdottir 1994). However, their enrichment during assimilation of some crustal components is also probable.

Figure $5 \mathrm{~B}$ shows that rhyolite is coeval with hydrothermal activity both in the northern and southern parts of the Tokaj Mts; however, the sampled northern rhyolite has no direct spatial connection to the hydrothermal fields. Thus, both spatial and temporal relationships between rhyolite occurrences considered in our study exist only in the southern Tokaj Mts. With the exception of one rhyolite and one perlite sample, the chlorine concentrations for the northern rhyolite are below $0.2 \mathrm{wt} \%$, whereas chlorine contents are above $0.2 \mathrm{wt} \%$ for the southern rhyolite. According to Webster (1997), a silicate liquid with $\mathrm{Cl}$ content below 0.2 wt $\%$ could not become saturated with respect to any magmatic volatile phase containing high amount of alkali-chlorides, which are necessary to form metalbearing hydrothermal solutions. Thus, it is highly possible that the southern rhyolite with higher $\mathrm{Cl}$ content could have generated hydrothermal 
mineralization and explain the presence of epithermal systems within and adjacent to rhyolite fields and volcanic structures in this part of the Tokaj Mts (Molnár et al. 1999).

\section{Conclusions}

On the basis of systematic sampling we established a new geochemical data base for the Sarmatian-Pannonian intermediate-acidic calc-alkaline volcanic rocks of the Tokaj Mts. The boron contents of rocks do not show any well-defined spatial relationships with the suspected subducted slab; thus, the direct influence of subduction-related components in the generation of magmas can be ruled out. We confirmed that the generation of magmas were connected to decompression melting of a metasomatized mantle during the relatively long-lived (13.4-9.4 Ma) volcanism of the Tokaj Mts. On the basis of B and Ba content of the studied rocks, the Altered Oceanic Crust-derived fluid influx is also obvious. Based on the above, the variation of the $\mathrm{Rb} / \mathrm{Zr}$ and $\mathrm{Ba} / \mathrm{Rb}$ ratios as well as the high negative $\mathrm{Eu}$ anomalies, together with the variation of the $\mathrm{La} / \mathrm{Yb}$ values of the rocks, suggests that both the assimilation of some crustal melts and the fractional crystallization affected the evolution of the magmas. In the northern part of the mountains, hydrothermal systems are not connected to rhyolite fields, and this may be related to the lower $\mathrm{Cl}$ content of these rocks. Rhyolite in the southern Tokaj Mts, where spatial and temporal relationships between the rhyolitic volcanism and hydrothermal activity is well established, is more Cl-rich.

\section{Acknowledgement}

The authors would like to thank to dr. Csaba Szabó and dr. János Földessy to their profound rewievs and also to Mr. Henry M. Lieberman for his remarks on the manuscript. This work was financially supported by the OTKA K68153 grant to Katalin Gméling and Zoltán Pécskay.

\section{References}

Anderson, D.L., Zs. Kasztovszky 2004: Application of PGAA with neutron beam. - In: Molnár, G.L.

(Ed.): Handbook of prompt gamma activation analysis with neutron beams. Kluwer Academic Publisher, pp. 148-152.

Bailey, E. H., K.V. Ragnarsdottir 1994: Uranium and thorium solubilities in subduction zone fluids. Earth Plan. Sci. Lett., 124, pp. 119-129.

Balogh, Kad. 1974: A kálium-argon kormeghatározási módszer alkalmazási lehetőségei és korlátai (The use and limintes of potassium-argon age determination method). - ATOMKI Bull., 16/4, pp. 373-387. (In Hungarian.)

Balogh, K., Z. Pécskay, V. Székyné-Fux, P. Gyarmati 1983: Chronology of Miocene volcanism in North-East Hungary. - Ann. Inst. Geol. Geofizica A, 61, 151 p.

Balogh, Kad. 1985: K/Ar dating of Neogene volcanic activity in Hungary: Experimental technique, experiences and methods of chronological studies. - ATOMKI Report, D/1, pp. 277-288. 
Belgya, T., Zs. Révay 2004: Gamma-ray spectrometry. - In: Molnár, G.L. (ed.): Handbook of prompt gamma activation analysis with neutron beams. Kluwer Academic Publisher, pp. 71-111.

Cameron, B. I., J.A. Walker, M.J. Carr, L.C. Patino, O. Matias, M.D. Feigenson 2003: Flux versus decompression melting at stratovolcanoes in southeastern Guatemala. - Jour. Volc. and Geot. Res., 119, pp. 21-50.

Csontos, L. 1995: Tertiary tectonic evolution of the intra-Carpathian area: a review. - Acta Volcanologica, 7/2, pp. 1-13.

Csontos, L., A. Nagymarosy, F. Horváth, M. Kovác 1992: Tertiary evolution of the intra-Carpathian area: a model. - Tectonophysics, 208, pp. 221-241.

Davidson, J., C. MacPherson, S. Turner 2007: Amphibole control in the differentiation of arc magmas. - Geochimica et Cosmochimica Acta, 71/15, A204-A204.

Downes, H., G. Pantó, T. Póka, D. Mattey, B. Greenwood 1995: Calc-alkaline volcanics of the Inner Carpathian arc, Northern Hungary: new geochemical and oxygen isotopic results. - In: Downes, H., O. Vaselli (Eds): Neogene and related magmatism in the Carpatho-Pannonian Region. Acta Vulcanologica, 7, pp. 29-41.

Gméling, K., Sz. Harangi, Zs. Kasztovszky 2005: Boron and chlorine concentration of volcanic rocks: an application of prompt gamma activation analysis. - J. Radioanal. Nucl. Chem., 11, pp. 265, pp. 201-214.

Gyarmati, P. 1977: Intermediate volcanism in the Tokaj Mountains. - Yearbook of the Hun. Nat. Geol. Ins. (MÁFI), 68, 196 p. (In Hungarian with English summary.)

Harangi, S., H. Downes, M. Thirlwall, K. Gméling 2007: Geochemistry, petrogenesis and geodynamic relationships of Miocene calc-alkalinevolcanic rocks in the Western Carpathian Arc, Eastern Central Europe. - Jour. of Petr., 48/12, pp. 2261-2287.

Horváth, F. 1988: Neotectonic behavior of the Alpine-Mediterranean Region. - In: Royden, L.H., F. Horváth (Eds): The Pannonian basin - A study in basin evolution. - Amer. Assoc. Petrol. Geol. Mem., 45, Tulsa, Okl., pp. 49-55.

Horváth, F, P. Dövényi, Á. Szalay, L.H. Royden 1988: Subsidence, thermal and maturation history of the Great Hungarian Plain. - In: Royden, L.H., F. Horváth (Eds): The Pannonian basin - A study in basin evolution. - Amer. Assoc. Petrol. Geol. Mem., 45, Tulsa, Okl., pp. 355-372.

Horváth, J., T. Fegyvári, T. Zelenka 1989: Paleovolcanic structures in the North-Tokaj Mountains interpreted on the basis of satellite imagery and aerial photography. - Acta Geologica Hungarica, 32/1-2, pp. 183-190.

Ilkeyné, Perlaki E. 1972: Acidic volcanism in the Tokaj Mountains. - Dissertation (In Hungarian.)

Ishikawa, T., F. Tera 1997: Source, composition and distribution of the fluid in the Kurile mantle wedge: Constraints from across-arc variations of $\mathrm{BrNb}$ and B isotopes. - Earth Plan. Sci. Lett., 152, pp. 123-138.

Kimura, J-I., T. Yoshida 2006: Contributions of Slab Fluid, Mantle Wedge and Crust to the Origin of Quaternary Lavas in the NE Japan - Arc. Jour. Pet., 47/11, pp. 2185-2232.

Kovács, I., Z. Zajacz, Cs. Szabó 2004: Type-II xenoliths and related metasomatism from the NógrádGömör Volcanic Field, Carpathian-Pannonian region (northern Hungary-southern Slovakia). Tectonophysics, 393, pp. 139-161.

Kovács, I., L. Csontos, Cs. Szabó, E. Bali, Gy. Falus, K. Benedek, Z. Zajacz 2007: Paleogene-early Miocene igneous rocks and geodynamics of the Alpine-Carpathian-Pannonian-Dinaric region: An integrated approach. - Geological Society of America Special Paper, 418, pp. 93-112.

Leeman, W.P., V.B. Sisson 1996: Geochemistry of boron and its implications for crustal and mantle processes. In: Grew, E.S., L.M. Anovitz (Eds): Reviews in Mineralogy, 33, Mineral. Soc. Am., Washington, DC, pp. 645-707.

Leeman, W.P., M.J. Carr, J.D. Morris 1994: Boron geochemistry of the Central American Volcanic Arc: Constraints on the genesis of subduction-related magmas. - Geoc. Cosm. Acta, 58, pp. 149-168.

Lexa, J., V. Konecny 1998: Geodynamic aspects of the Neogene to Quaternary volcanism. - In: Rakus, M. (Ed.): Geodynamic Development of the Western Carpathians. Bratislava: Geological Survey of Slovak Republic, pp. 219-240. 
Liffa, A. 1928: Jelentés Fony és Regéc környékéről (Report from the Fony-Regéc area). - Ann. Rep. Hun. Nat. Geol Inst., 1924, pp. 15-17. (In Hungarian.)

Molnár, F, T. Zelenka, E. Mátyás, Z. Pécskay, B. Bajnóczi, J. Kiss, I. Horváth 1999: Epithermal mineralization of the Tokaj Mountains, Northeast Hungary: Shallow levels of low-sulfidisation type systems. - In: Molnár, F, J. Lexa, J.W. Hedenquist (Eds): Epithermal mineralization of the Western Carpathians. Guidebook for the Society of Economic Geologists Field Conference, 4-13. September, pp. 109-153.

Moyen, J-F. 2009: High Sr/Y and La/Yb ratios: The meaning of the "adakitic signature". - Lithos, 112, pp. 556-574.

Nemcok, M., L. Pospíšil, J. Lexa, R.A. Donelick 1998: Tertiary subduction and slab break-off model of the Carpathian-Pannonian region. - Tectonophysics, 295, pp. 307-340.

Németh, K., Z. Pécskay, U. Martin, K. Gméling, F. Molnár, S. Cronin 2006: Peperites and soft sediment deformation textures of a shallow subaqueous Miocene rhyolitic cryptodome and dyke complex, Pálháza, Hungary. LASI-II. Physical geology of subvolcanic systems: Laccoliths, sills and dykes: 36 .

Pálfy, M. 1915: A Pálháza környéki riolitterület Abaúj-Torna vármegyében (The rhyolite area near Pálháza in Abaúj-Tolna county). - Ann. Rep. Hun. Nat. Geol, Inst., 1914, pp. 312-323. (In Hungarian.)

Pálfy, M. 1916: Az erupciós kőzetek zöldkövesedése ("Greenstoning" of eruptive rocks). - Bull. Hun. Nat. Geol. Inst., 46/4-6, pp. 73-85. (In Hungarian.)

Pálfy, M. 1935: Adatok a Tokaji-hegység harmadkori erupcióinak korviszonyaihoz (Data to the eruption age relations of the Tertiary of the Tokaj Mts). - Bull. Hun. Nat. Geol. Inst, 57, pp. 67-71. (In Hungarian.)

Peccerillo, A. 2005: Plio-Quaternary Volcanism in Italy: Petrology, geochemistry, geodynamics. Berlin, Springer.

Pécskay, Z., F. Molnár 2002: Relationships between volcanism and hydrothermal activity in the Tokaj Mountains, Northeast Hungary, based on K-Ar Ages. - Geologica Carpathica, 53/5, pp. 303-314.

Pécskay, Z., K. Balogh, V. Székyné-Fux, P. Gyarmati 1986: Geochronological investigations on the Neogene volcanism of the Tokaj Mountains. - SCI Geologicky Zbornik, Geol. Carpath., 37, pp. 635-655.

Pécskay, Z., K. Balogh, V. Székyné Fux, P. Gyarmati 1987: Geochronological investigations of the Neogene volcanism of Tokaj Mts. (A Tokaji-hegység miocén vulkánosságának K/Ar geokronológiája) - Bull. Hung. Nat. Geol. Soc., 117, pp. 237-253. (In Hungarian.)

Pécskay, Z., J. Lexa, A. Szakács, Kad. Balogh, I. Seghedi, V. Konecný, M. Kovác, E. Márton, V. SzékyFux, T. Póka, P. Gyarmaty, O. Edelstein, E. Rosu, B. Zec 1995: Space and time distribution of Neogene-Quaternary volcanism in the Carpatho-Pannonian region. - Acta Volcanologica, 72, pp. 15-29.

Pécskay, Z., J. Lexa, A. Szakács, I. Seghedi, K. Balogh, V. Konecný, T. Zelenka, M. Kovacs, T. Póka, A. Fülöp, E. Márton, C. Panaiotu, V. Cvetkovic 2006: Geochronology of Neogene magmatism in the Carpathian arc and intra-Carpathian area. - Geologica Carpathica, 57, pp. 511-530.

Pentelényi, L. 1972: Explanation to the geological map of the Tokaj Mts., 1:25 000 Füzérradvány-Széphalom (Magyarázó a Tokaji-hegység 1:25 000 méretarányú térképsorozatához. Füzérradvány-Széphalom), Published by the Hun. Nat. Geol. Inst. (In Hungarian.)

Révay, Zs., T. Belgya, P.P. Ember, G.L. Molnár 2001: Recent developments in Hypermet-PC. - J. Radioanal. Nucl. Chem., 248, pp. 401-405.

Révay, Zs. 2009: Determining elemental composition using prompt gamma activation analysis. Anal. Chem., 81, pp. 6851-6859.

Ryan, J.G., J. Morris, F. Tera, D.H. Leeman, A. Tsvetkov 1995: Cross-arc geochemical variations in the Kurile arc as a function of slab depth. - Science, 270, pp. 625-627. 
Sano, T., T. Hasenaka, A. Shimaoka, C. Yonezawa, T. Fukuoka 2001: Boron contents of Japan Trench sediments and Iwate basaltic lavas, Northeast Japan arc: estimation of sediment-derived fluid contribution in mantle wedge. - Earth Plan. Sci. Lett., 186, pp. 187-198.

Seghedi, I., H. Downes, A. Szakács, P.R.D. Mason, M.F. Thirlwall, E. Rosu, Z. Pécskay, E. Márton, C. Panaiotu 2004: Neogene-Quaternary magmatism and geodynamics in the

Carpathian-Pannonian region: a synthesis. - Lithos, 72, pp. 117-146.

Simkó, Gy. 1927: A Tokaji-Nagyhegy effuzív kőzeteinek litoklázisrendszere és ennek morfológiai szerepe (The crack-system and its morphological rule of the effusive rocks of the TokajNagyhegy). - Bull. Hun. Nat. Geol. Inst, 57, pp. 12-20. (In Hungarian.)

Steiger, R.H., E. Jägger 1977: Subcomisson on Geochronology: Convention on the use of decay constant in geology and geochronology. - Earth. Plan. Sci. Lett., 36/3, pp. 359-362.

Sun, S., W.F. McDonough 1989: Chemical and isotopic systematics of oceanic basalts: implications for mantle compositions and processes. - In: Saunders, A.D., M.J. Norry (Eds): Magmatism in the Ocean Basins. Spec. Publ. Geol. Soc., 42, pp. 313-345.

Szabó, Cs., Sz. Harangi, L. Csontos 1992: Review of Neogene and Quaternary volcanism of the Carpathian-Pannonian region. - Tectonophysics, 208, pp. 243-256.

Szabó, J. 1873: A trachytok osztályozása természetes rendszer szerint (Natural classification of trachites). - Bull. Hun. Nat. Geol. Inst., 3, pp. 8-20. (In Hungarian.)

Szabó, J. 1881: A trachytok makrográfiai osztályozása (Macroscopic classification of trachites). - Bull. Hun. Nat. Geol. Inst., 11, pp. 209-219. (In Hungarian.)

Szádeczky, Gy. 1897: Sátoralja-Újhelytől északnyugatra Ruda-Bányácska és Kovácsvágás közé eső terület geológiai és kőzettani tekintetben (Geological and petrological aspects of the RudaBányácska-Kovácsvágás area, northeast from Sátoralja-Újhely). - Bull. Hun. Nat. Geol. Inst., 27, pp. 273-326. (In Hungarian.)

Széky-Fux, V. 1970: The Telkibánya mineralization and its Intra-Carpathian connections (Telkibányai ércesedése és kárpáti kapcsolatai). - Akadémiai Kiadó, Budapest. 266 p. (In Hungarian with English abstract.)

Taylor, H.P.J. 1980: The effects of assimilation of country rocks by magmas on $18 \mathrm{O} / 16 \mathrm{O}$ and $87 \mathrm{Sr} / 86 \mathrm{Sr}$ systematics in igneous rocks. - Earth Plan. Sci. Lett., 47, pp. 243-254.

Taylor, S.R., S.M. McLennan 1985: The Continental Crust; Its composition and evolution; an examination of the geochemical record preserved in sedimentary rocks. - Blackwell, Oxford, $312 \mathrm{p}$.

Tonarini, S., W.P. Leeman, G. Ferrara 2001: Boron isotopic variations in lavas of the Aeolian volcanic arc, South Italy. - Jour. Volc. and Geot. Res., 110, pp. 155-170.

Tonarini, S., W.P. Leeman, L. Civetta, M. D'Antonio, G. Ferrara, A. Necco 2004: B/Nb and 11B systematics in the Phlegrean Volcanic District, Italy. - Jour. Volc. and Geot. Res., 133, pp. 123-139.

Webster, J.D. 1997: Chloride solubility in felsic melts and the role of chloride in magmatic degassing. - Jour. Petr., 38/12, pp. 1793-1807.

Zelenka, T. 1963: Tokajhegyalja DNY-i részének földtani felépítése (Geological structure of the southwestern part of Tokajhegyalja). - Doctorate dissertation. (In Hungarian.) 